ALEA, Lat. Am. J. Probab. Math. Stat. 15, 1183-1213 (2018)

DOI: $10.30757 /$ ALEA.v15-44

\title{
Local Skorokhod topology on the space of cadlag processes
}

\author{
Mihai Gradinaru and Tristan Haugomat \\ Université de Rennes, CNRS, IRMAR - UMR 6625 F-35000 Rennes, France. \\ E-mail address: \{mihai.gradinaru, tristan.haugomat \} Quniv-rennes1.fr
}

\begin{abstract}
We modify the global Skorokhod topology, on the space of cadlag paths, by localising with respect to space variable, in order to include the eventual explosions. The tightness of families of probability measures on the paths space endowed with this local Skorokhod topology is studied and a characterization of Aldous type is obtained. The local and global Skorokhod topologies are compared by using a time change transformation.
\end{abstract}

\section{Introduction}

The study of cadlag Lévy-type processes has been an important challenge during the last twenty years. This was due to the fact that phenomena like jumps and unbounded coefficients of characteristic exponent (or symbol) should be taken in consideration in order to get more realistic models.

To perform a systematic study of this kind of trajectories one needs, on one hand, to consider the space of cadlag paths with some appropriate topologies, e.g. Skorokhod's topologies. On the other hand, it was a very useful observation that a unified manner to tackle a lot of questions about large classes of processes is the martingale problem approach. Identifying tightness is an important step when studying sequences of distributions of processes solving associated martingale problems and the Aldous criterion is one of the most employed.

The martingale problem approach was used for several situations: diffusion processes, stochastic differential equations driven by Brownian motion, Lévy processes, Poisson random measures (see, for instance, Stroock, 1975, Stroock and Varadhan, 2006, Kurtz, 2011...). Several technical hypotheses (for instance, entire knowledge of the generator, bounded coefficients hypothesis, assumptions concerning explosions...) provide some limitation on the conclusions of certain results, in particular, on convergence results.

Received by the editors February 2nd, 2018; accepted September 10th, 2018.

2010 Mathematics Subject Classification. 60B10, 60J75, 54E70, 54A10.

Key words and phrases. cadlag processes, explosion time, local Skorokhod topology, Aldous tightness criterion, time change transformation. 
The present paper constitutes our first step in studying Markov processes with explosion and, in particular in the martingale problem setting. It contains the study of the so-called local Skorokhod topology and of a time change transformation of cadlag paths. The detailed study of the martingale problem, of Lévy-type processes and several applications will be presented elsewhere (see Gradinaru and Haugomat, 2017b and Gradinaru and Haugomat, 2017a).

One of our motivations is that we wonder whether the solution of a well-posed martingale problem is continuous with respect to the initial distribution. The classical approach when one needs to take into consideration the explosion of the solution is to compactify the state space by one point, say $\Delta$, and to endow the cadlag paths space by the Skorokhod topology (see, for instance, Ethier and Kurtz, 1986, Kallenberg, 2002). Unfortunately, this usual topology is not appropriate when we relax hypotheses on the martingale problem setting.

The simplest example is provided by the differential equation

$$
\dot{x}_{t}=b\left(t, x_{t}\right), \quad t>0, \quad \text { starting from } x_{0} \in \mathbb{R}^{d},
$$

where $b: \mathbb{R}_{+} \times \mathbb{R}^{d} \rightarrow \mathbb{R}^{d}$ is a locally Lipschitz function. The unique maximal solution exists by setting $x_{t}=\Delta$, after the explosion time. In general, for some $t>0$, the mapping $x_{0} \mapsto x_{t}$ is not continuous, and in particular $x_{0} \mapsto x_{\bullet}$ is not continuous for the usual (global) Skorokhod topology. As an illustration, let us consider

$$
\dot{x}_{t}=(1-t) x_{t}^{2}, \quad t>0, \quad x_{0} \in \mathbb{R} .
$$

For any initial condition $x_{0}$, the unique maximal solution is given by

$$
x_{t}=\left(\frac{t^{2}}{2}-t+\frac{1}{x_{0}}\right)^{-1} \quad \text { before } \quad t_{\max }= \begin{cases}\infty, & \text { if } x_{0} \in[0,2), \\ 1-\sqrt{1-2 / x_{0}}, & \text { if } x_{0} \geq 2, \\ 1+\sqrt{1-2 / x_{0}}, & \text { if } x_{0}<0,\end{cases}
$$

and $x_{t}:=\Delta$, after $t_{\max }$. Indeed, this trajectory is not continuous with respect to the initial condition in the neighbourhood of $x_{0}=2$. To achieve the continuity of the mapping $x_{0} \mapsto x_{\bullet}$ our idea will be to localise the topology on the paths space, not only with respect to the time variable but also with respect to the space variable. More precisely, we need to consider uniform convergence until the exit time from some compact subset of $\mathbb{R}_{+} \times \mathbb{R}^{d}$.

We adapt this idea to cadlag paths by following a similar approach as in Billingsley (1999) and we get the local Skorokhod topology which is weaker than the usual (global) Skorokhod topology. Then, we describe the compactness and the tightness in connection with this topology. Furthermore, we state and prove a version of the Aldous criterion, which is an equivalence, as in Rebolledo (1979).

As in Ethier and Kurtz (1986), pp. 306-311, we introduce a time change transformation of the cadlag path $x$ by the positive continuous function $g$. Roughly speaking, it is defined by $(g \cdot x)_{t}:=x_{\tau_{t}}$ with $\tau_{t}$ the unique solution starting from 0 of the equation $\dot{\tau}_{t}=g\left(x_{\tau_{t}}\right)$. Another novelty of our paper is the employ of the time change transformation to compare the local Skorokhod topology with the usual (global) Skorokhod topology.

Our paper is organised as follows: the following section is mainly devoted to the study of the local Skorokhod topology on spaces of cadlag paths: the main result is a tightness criterion. Properties of the time change mapping, in particular the continuity, and the connection between the local and global Skorokhod topologies 
are described in Section 3. The last section contains technical proofs, based on local Skorokhod metrics, of results stated in Section 2.

\section{Paths spaces}

2.1. Local spaces of cadlag paths. Let $S$ be a locally compact Hausdorff space with countable base. This topological feature is equivalent with the fact that $S$ is separable and can be endowed by a metric for which the unit balls are compact, so, $S$ is a Polish space. Take $\Delta \notin S$, and we will denote by $S^{\Delta} \supset S$ the one-point compactification of $S$, if $S$ is not compact, or the topological sum $S \sqcup\{\Delta\}$, if $S$ is compact (so, $\Delta$ is an isolated point). Clearly, $S^{\Delta}$ is a compact Hausdorff space with countable base which could be also endowed with a metric. This latter metric will be used to construct various useful functions, compact and open subsets.

For any topological space $A$ and any subset $B \subset \mathbb{R}$, we will denote by $\mathrm{C}(A, B)$ the set of continuous functions from $A$ to $B$, and by $\mathrm{C}_{b}(A, B)$ its subset of bounded continuous functions. We will abbreviate $\mathrm{C}(A):=\mathrm{C}(A, \mathbb{R})$ and $\mathrm{C}_{b}(A):=\mathrm{C}_{b}(A, \mathbb{R})$. All along the paper we will denote $C \Subset A$ for a subset $C$ which is compactly embedded in $A$. Similarly, $C \notin A$ means either that $C$ is not a subset of $A$ or $C$ is not compactly embedded in $A$.

We start with the definition of our spaces of trajectories:

Definition 2.1 (Spaces of cadlag paths). Define the space of exploding cadlag paths

$$
\mathbb{D}_{\exp }(S):=\left\{\begin{array}{l|l}
x:[0, \xi(x)) \rightarrow S & \begin{array}{l}
0 \leq \xi(x) \leq \infty \\
\forall t_{0} \in[0, \xi(x)) \\
\forall t_{0} \in(0, \xi(x)) \quad x_{t_{0}}=\lim _{t \downarrow t_{0}} x_{t}, \\
\forall=\lim _{t \uparrow t_{0}} x_{t} \text { exists in } S
\end{array}
\end{array}\right\} .
$$

When $\xi(x)=0$, we assume that $x$ is the empty subset of $[0,0) \times S$. For a path $x$ from $\mathbb{D}_{\exp }(S), \xi(x)$ is its lifetime or explosion time. We identify $\mathbb{D}_{\exp }(S)$ with a subset of $\left(S^{\Delta}\right)^{\mathbb{R}_{+}}$by using the mapping

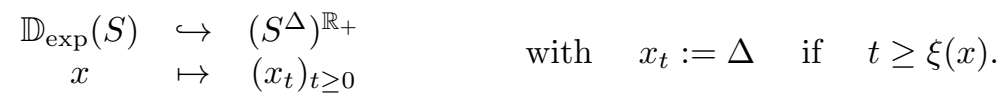

We define the local cadlag space as the subspace

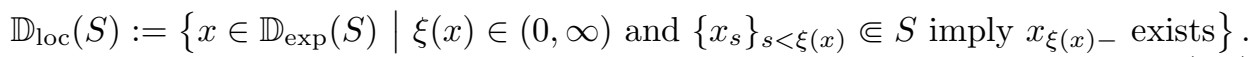

We also introduce the global cadlag space as the subspace of $\mathbb{D}_{\text {loc }}(S)$

$$
\mathbb{D}(S):=\left\{x \in \mathbb{D}_{\text {loc }}(S) \mid \xi(x)=\infty\right\} \subset S^{\mathbb{R}_{+}} .
$$

We will always denote by $X$ the canonical process on $\mathbb{D}_{\exp }(S), \mathbb{D}_{\text {loc }}(S)$ and $\mathbb{D}(S)$ without danger of confusion. We endow each of $\mathbb{D}_{\exp }(S), \mathbb{D}_{\text {loc }}(S)$ and $\mathbb{D}(S)$ with a $\sigma$-algebra $\mathcal{F}:=\sigma\left(X_{s}, 0 \leq s<\infty\right)$ and a filtration $\mathcal{F}_{t}:=\sigma\left(X_{s}, 0 \leq s \leq t\right)$. We will always skip the argument $X$ for the explosion time $\xi(X)$ of the canonical process.

The following result provides a useful class of measurable mappings:

Proposition 2.2. For $t_{0} \in \mathbb{R}_{+}$, the mapping

$$
\begin{array}{ccc}
\mathbb{D}_{\exp }(S) \times\left[0, t_{0}\right] & \rightarrow S^{\Delta} \\
(x, t) & \mapsto x_{t}
\end{array}
$$


is $\mathcal{F}_{t_{0}} \otimes \mathcal{B}\left(\left[0, t_{0}\right]\right)$-measurable. For $t_{0} \in \mathbb{R}_{+}^{*}$, the set

$$
A:=\left\{(x, t) \in \mathbb{D}_{\exp }(S) \times\left(0, t_{0}\right] \mid x_{t-} \text { exists in } S^{\Delta}\right\}
$$

belongs to $\mathcal{F}_{t_{0}-} \otimes \mathcal{B}\left(\left(0, t_{0}\right]\right)$ and the mapping

$$
\begin{array}{cl}
A & \rightarrow S^{\Delta} \\
(x, t) & \mapsto x_{t-}
\end{array}
$$

is $\mathcal{F}_{t_{0}-} \otimes \mathcal{B}\left(\left(0, t_{0}\right]\right)$-measurable. For $U$ an open subset of $S$ and for $t_{0} \in \mathbb{R}_{+}$, the set

$$
B:=\left\{(x, s, t) \in \mathbb{D}_{\exp }(S) \times\left[0, t_{0}\right]^{2} \mid\left\{x_{u}\right\}_{s \wedge t \leq u<s \vee t} \Subset U\right\}
$$

belongs to $\mathcal{F}_{t_{0}-} \otimes \mathcal{B}\left(\left[0, t_{0}\right]\right)^{\otimes 2}$ and the mapping

$$
\begin{aligned}
B \times \mathrm{C}(U) & \rightarrow \mathbb{R} \\
(x, s, t, h) & \mapsto \int_{s}^{t} h\left(x_{u}\right) \mathrm{d} u
\end{aligned}
$$

is $\mathcal{F}_{t_{0}-} \otimes \mathcal{B}\left(\left[0, t_{0}\right]\right)^{\otimes 2} \otimes \mathcal{B}(\mathrm{C}(U))$-measurable.

Before proving this proposition we state a corollary which give a useful class of stopping times:

Corollary 2.3. For any $\left(\mathcal{F}_{t}\right)$-stopping time $\tau_{0}, \mathcal{U}$ an open subset of $S^{2}, h \in$ $\mathrm{C}\left(\mathcal{U}, \mathbb{R}_{+}\right)$a continuous function and $M: \mathbb{D}_{\exp }(S) \rightarrow[0, \infty]$ a $\mathcal{F}_{\tau_{0}}$-measurable map, the mapping

$$
\tau:=\inf \left\{t \geq \tau_{0} \mid\left\{\left(X_{\tau_{0}}, X_{s}\right)\right\}_{\tau_{0} \leq s \leq t} \notin \mathcal{U} \text { or } \int_{\tau_{0}}^{t} h\left(X_{\tau_{0}}, X_{s}\right) \mathrm{d} s \geq M\right\}
$$

is a $\left(\mathcal{F}_{t}\right)$-stopping time. In particular, $\xi$ is a stopping time. Furthermore, if $U \subset S$ is an open subset,

$$
\tau^{U}:=\inf \left\{t \geq 0 \mid X_{t-} \notin U \text { or } X_{t} \notin U\right\} \leq \xi
$$

is a stopping time.

Proof of Corollary 2.3: For each $t \geq 0$, using Proposition 2.2 it is straightforward to obtain that

$$
Y:= \begin{cases}-1 & \text { if } \tau_{0}>t, \\ \int_{\tau_{0}}^{t} h\left(X_{\tau_{0}}, X_{s}\right) \mathrm{d} s & \text { if } \tau_{0} \leq t \text { and }\left\{\left(X_{\tau_{0}}, X_{s}\right)\right\}_{\tau_{0} \leq s \leq t} \Subset \mathcal{U}, \\ \infty & \text { otherwise. }\end{cases}
$$

is $\mathcal{F}_{t}$-measurable. Hence

$$
\{\tau \leq t\}=\{Y \geq M\}=\{Y \geq M\} \cap\left\{\tau_{0} \leq t\right\} \in \mathcal{F}_{t},
$$

so, $\tau$ is a $\left(\mathcal{F}_{t}\right)$-stopping time.

Proof of Proposition 2.2: Let $d$ be a complete metric for the topology of $S$, note that

$$
A=\bigcap_{\varepsilon \in \mathbb{Q}_{+}^{*}} \bigcup_{\delta \in \mathbb{Q}_{+}^{*}} \bigcap_{q_{1}, q_{2} \in \mathbb{Q}_{+} \cap\left[0, t_{0}\right)}\left\{q_{1}, q_{2} \in[t-\delta, t) \Rightarrow d\left(x_{q_{1}}, x_{q_{2}}\right) \leq \varepsilon\right\}
$$

so, $A$ belongs to $\mathcal{F}_{t_{0}-} \otimes \mathcal{B}\left(\left(0, t_{0}\right]\right)$. It is clear that for each $n \in \mathbb{N}$

$$
\begin{array}{ccc}
A & \rightarrow & S^{\Delta} \\
(x, t) & \mapsto & x_{\frac{t_{0}}{n+1}\left\lfloor\frac{n t}{t_{0}}\right\rfloor}
\end{array}
$$


is $\mathcal{F}_{t_{0}-} \otimes \mathcal{B}\left(\left(0, t_{0}\right]\right)$-measurable, where $\lfloor r\rfloor$ denotes the integer part of the real number $r$. Letting $n \rightarrow \infty$ we obtain that $(x, t) \mapsto x_{t-}$ is $\mathcal{F}_{t_{0}-} \otimes \mathcal{B}\left(\left(0, t_{0}\right]\right)$-measurable. The proof is similar for $(x, t) \mapsto x_{t}$. To prove that $B$ is measurable, let $\left(K_{n}\right)_{n \in \mathbb{N}}$ be an increasing sequence of compact subsets of $U$ such that $U=\bigcup_{n} K_{n}$. Then

$$
\begin{aligned}
B & =\bigcup_{n \in \mathbb{N}}\left\{(x, s, t) \in \mathbb{D}_{\exp }(S) \times\left[0, t_{0}\right]^{2} \mid\left\{x_{u}\right\}_{s \wedge t \leq u<s \vee t} \subset K_{n}\right\} \\
& =\bigcup_{n \in \mathbb{N}} \bigcap_{\substack{q \in \mathbb{Q}_{+} \\
q<t_{0}}}\left\{(x, s, t) \in \mathbb{D}_{\exp }(S) \times\left[0, t_{0}\right]^{2} \mid s \wedge t \leq q<s \vee t \Rightarrow x_{q} \in K_{n}\right\},
\end{aligned}
$$

so, $B \in \mathcal{F}_{t_{0}-} \otimes \mathcal{B}\left(\left[0, t_{0}\right]\right)^{\otimes 2}$. To verify the last part, let us note that for $n \in \mathbb{N}^{*}$ the mapping from $B \times \mathrm{C}(U)$

$$
(x, s, t, h) \mapsto \frac{\operatorname{sign}(t-s)}{n} \sum_{i=0}^{n-1} h\left(x_{\frac{i t_{0}}{n}}\right) \mathbb{1}_{s \wedge t \leq \frac{i t_{0}}{n}<s \vee t}
$$

is $\mathcal{F}_{t_{0}-} \otimes \mathcal{B}\left(\left[0, t_{0}\right]\right)^{\otimes 2} \otimes \mathcal{B}(\mathrm{C}(U))$-measurable. Here and elsewhere we denote by $\mathbb{N}^{*}=\mathbb{N} \backslash\{0\}$ the set of positive entire numbers. Letting $n \rightarrow \infty$, the same thing is true for the mapping

$$
\begin{aligned}
B \times \mathrm{C}(U) & \rightarrow \mathbb{R} \\
(x, s, t, h) & \mapsto \int_{s}^{t} h\left(x_{u}\right) \mathrm{d} u .
\end{aligned}
$$

2.2. Local Skorokhod topology. To simplify some statements, in this section we will consider a metric $d$ on $S$. To describe the convergence of a sequence $\left(x^{k}\right)_{k \in \mathbb{N}} \subset$ $\mathbb{D}_{\text {loc }}(S)$ for our topology on $\mathbb{D}_{\text {loc }}(S)$, we need to introduce the following two spaces: we denote by $\Lambda$ the space of increasing bijections from $\mathbb{R}_{+}$to $\mathbb{R}_{+}$, and by $\widetilde{\Lambda} \subset \Lambda$ the space of increasing bijections $\lambda$ with $\lambda$ and $\lambda^{-1}$ locally Lipschitz. For $\lambda \in \Lambda$ and $t \in \mathbb{R}_{+}$we denote

$$
\|\lambda-\mathrm{id}\|_{t}:=\sup _{0 \leq s \leq t}\left|\lambda_{s}-s\right|=\left\|\lambda^{-1}-\mathrm{id}\right\|_{\lambda_{t}} .
$$

For $\lambda \in \widetilde{\Lambda}$, let $\dot{\lambda} \in \mathrm{L}_{\mathrm{loc}}^{\infty}(\mathrm{d} s)$ be the density of $\mathrm{d} \lambda$ with respect to the Lebesgue measure. This density is non-negative and locally bounded below, and for $t \in \mathbb{R}_{+}$ denote

$$
\|\log \dot{\lambda}\|_{t}:=\operatorname{esssup}_{0 \leq s \leq t}\left\|\log \dot{\lambda}_{s}\right\|=\sup _{0 \leq s_{1}<s_{2} \leq t}\left|\log \frac{\lambda_{s_{2}}-\lambda_{s_{1}}}{s_{2}-s_{1}}\right|=\left\|\log \left(\frac{\mathrm{d} \lambda_{s}^{-1}}{\mathrm{~d} s}\right)\right\|_{\lambda_{t}} .
$$

The proofs of the following theorems use the strategy developed in $§ 12$, pp. 121-137 from Billingsley (1999), and are postponed to Section 4.

Theorem 2.4 (Local Skorokhod topology). There exists a unique Polish topology on $\mathbb{D}_{\text {loc }}(S)$, such that a sequence $\left(x^{k}\right)_{k \in \mathbb{N}}$ converges to $x$ for this topology if and only if there exists a sequence $\left(\lambda^{k}\right)_{k \in \mathbb{N}}$ in $\Lambda$ such that

- either $\xi(x)<\infty$ and $\left\{x_{s}\right\}_{s<\xi(x)} \Subset S: \lambda_{\xi(x)}^{k} \leq \xi\left(x^{k}\right)$ for $k$ large enough and $\sup _{s<\xi(x)} d\left(x_{s}, x_{\lambda_{s}^{k}}^{k}\right) \longrightarrow 0, \quad \quad x_{\lambda_{\xi(x)}^{k}}^{k} \longrightarrow \Delta, \quad\left\|\lambda^{k}-\mathrm{id}\right\|_{\xi(x)} \longrightarrow 0, \quad$ as $k \rightarrow \infty$, 
- or $\xi(x)=\infty$ or $\left\{x_{s}\right\}_{s<\xi(x)} \notin S$ : for all $t<\xi(x)$, for $k$ large enough $\lambda_{t}^{k}<\xi\left(x^{k}\right)$ and

$$
\sup _{s \leq t} d\left(x_{s}, x_{\lambda_{s}^{k}}^{k}\right) \longrightarrow 0, \quad\left\|\lambda^{k}-\mathrm{id}\right\|_{t} \longrightarrow 0, \quad \text { as } k \rightarrow \infty .
$$

This topology is also described by a similar characterisation with $\lambda^{k} \in \Lambda$ and $\| \lambda^{k}-$ id\| replaced, respectively, by $\lambda^{k} \in \widetilde{\Lambda}$ and $\left\|\log \dot{\lambda}^{k}\right\|$. Moreover, the Borel $\sigma$-algebra $\mathcal{B}\left(\mathbb{D}_{\text {loc }}(S)\right)$ coincides with the $\sigma$-algebra $\mathcal{F}$.

Definition 2.5. The topology on $\mathbb{D}_{\text {loc }}(S)$ whose existence is stated in the latter theorem will be called the local Skorokhod topology. The trace topology from $\mathbb{D}_{\text {loc }}(S)$ to $\mathbb{D}(S)$ will be called the global Skorokhod topology.

Remark 2.6. 1) We point out that these topologies do not depend on the metric $d$ of $S$ and this is a consequence of the fact that two metrics on a compact set are uniformly equivalent (cf. Lemma 4.3 below).

2) The convergence conditions of Theorem 2.4 may be summarised as: a sequence $\left(x_{k}\right)_{k}$ converges to $x$ for the local Skorokhod topology if and only if there exists a sequence $\left(\lambda^{k}\right)_{k}$ in $\Lambda$ satisfying that for any $t \in \mathbb{R}_{+}$such that $\left\{x_{s}\right\}_{s<t} \Subset S$, for $k$ large enough $\lambda_{t}^{k} \leq \xi\left(x^{k}\right)$ and

$$
\sup _{s<t} d\left(x_{s}, x_{\lambda_{s}^{k}}^{k}\right) \longrightarrow 0, \quad x_{\lambda_{t}^{k}}^{k} \longrightarrow x_{t}, \quad\left\|\lambda^{k}-\mathrm{id}\right\|_{t} \longrightarrow 0, \quad \text { as } k \rightarrow \infty .
$$

3) A sequence $\left(x^{k}\right)_{k}$ from $\mathbb{D}(S)$ converges to $x \in \mathbb{D}(S)$ for the global Skorokhod topology if and only if there exists a sequence $\left(\lambda^{k}\right)_{k}$ in $\Lambda$ such that for all $t \geq 0$

$$
\sup _{s \leq t} d\left(x_{s}, x_{\lambda_{s}^{k}}^{k}\right) \longrightarrow 0, \quad\left\|\lambda^{k}-\mathrm{id}\right\|_{t} \longrightarrow 0, \quad \text { as } k \rightarrow \infty .
$$

In fact, we recover the usual Skorokhod topology on $\mathbb{D}(S)$, which is described, for instance, in $§ 16$ pp. 166-179 from Billingsley (1999).

We are now interested to characterise the sets of $\mathbb{D}_{\text {loc }}(S)$ which are compact and also to obtain a criterion for the tightness of a subset of probability measures in $\mathcal{P}\left(\mathbb{D}_{\text {loc }}(S)\right)$. For $x \in \mathbb{D}_{\exp }(S), t \geq 0, K \subset S$ compact and $\delta>0$, define

$$
\omega_{t, K, x}^{\prime}(\delta):=\inf \left\{\begin{array}{l|l}
\sup _{\substack{0 \leq i<N \\
t_{i} \leq s_{1}, s_{2}<t_{i+1}}} d\left(x_{s_{1}}, x_{s_{2}}\right) & \begin{array}{l}
N \in \mathbb{N}, 0=t_{0}<\cdots<t_{N} \leq \xi(x) \\
\left(t_{N}, x_{t_{N}}\right) \notin[0, t] \times K \\
\forall 0 \leq i<N: t_{i+1}-t_{i}>\delta
\end{array}
\end{array}\right\} .
$$

We give some properties of $\omega^{\prime}$.

\section{Proposition 2.7.}

i) Consider $x \in \mathbb{D}_{\exp }(S)$. Then $x$ belongs to $\mathbb{D}_{\text {loc }}(S)$ if and only if

$$
\forall t \geq 0, \forall K \subset S \text { compact }, \quad \omega_{t, K, x}^{\prime}(\delta) \underset{\delta \rightarrow 0}{\longrightarrow} 0 .
$$

ii) For all $t \geq 0, K \subset S$ compact and $\delta>0$, the mapping

$$
\begin{array}{ccc}
\mathbb{D}_{l o c}(S) & \rightarrow & {[0,+\infty]} \\
x & \mapsto & \omega_{t, K, x}^{\prime}(\delta)
\end{array}
$$

is upper semi-continuous. 
Proof: Suppose that $x \in \mathbb{D}_{\text {loc }}(S)$ and let $t \geq 0$ be and consider a compact set $K \subset S$. There exists $T \leq \xi(x)$ such that $\left(T, x_{T}\right) \notin[0, t] \times K$ and the limit $x_{T-}$ exists in $S$. Let $\varepsilon>0$ be arbitrary and consider $I$ the set of times $s \leq T$ for which there exists a subdivision

$$
0=t_{0}<\cdots<t_{N}=s
$$

such that

$$
\sup _{\substack{0 \leq i<N \\ t_{i} \leq s_{1}, s_{2}<t_{i+1}}} d\left(x_{s_{1}}, x_{s_{2}}\right) \leq \varepsilon
$$

It is clear that $I$ is an interval of $[0, T]$ containing 0 : set $t^{*}:=\sup I$. Since there is existence of the limit $x_{t^{*}-}$, then $t^{*} \in I$, and, since $x$ is right-continuous, $t^{*}=T$. Hence, $T \in I$ and there exists $\delta>0$ such that $\omega_{t, K, x}^{\prime}(\delta) \leq \varepsilon$.

Conversely, let's take $x \in \mathbb{D}_{\exp }(S)$ such that $\xi(x)<\infty,\left\{x_{s}\right\}_{s<\xi(x)} \Subset S$ and

$$
\forall t \geq 0, \forall K \subset S \text { compact, } \omega_{t, K, x}^{\prime}(\delta) \underset{\delta \rightarrow 0}{\longrightarrow} 0 .
$$

We need to prove that the limit $x_{\xi(x)-}$ exists in $S$. Let $y_{1}, y_{2}$ be any two limits points of $x_{s}$, as $s \rightarrow \xi(x)$. We will prove that $y_{1}=y_{2}$. Let $\varepsilon>0$ be arbitrary. By

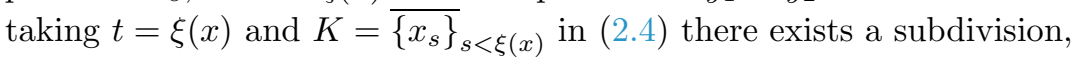

$$
0=t_{0}<\cdots<t_{N}=\xi(x),
$$

such that

$$
\sup _{\substack{0 \leq i<N \\ t_{i} \leq s_{1}, s_{2}<t_{i+1}}} d\left(x_{s_{1}}, x_{s_{2}}\right) \leq \varepsilon .
$$

Replacing in the latter inequality the two sub-sequences tending toward $y_{1}, y_{2}$, we can deduce that $d\left(y_{1}, y_{2}\right) \leq \varepsilon$, and letting $\varepsilon \rightarrow 0$ we get $y_{1}=y_{2}$.

We proceed with the proof of part ii). Let $\left(x^{k}\right)_{k} \subset \mathbb{D}_{\text {loc }}(S)$ be such that $x^{k}$ converges to $x \in \mathbb{D}_{\text {loc }}(S)$ and let $\left(\lambda^{k}\right)_{k} \subset \widetilde{\Lambda}$ be such in Theorem 2.4. We need to prove that,

$$
\limsup _{k \rightarrow \infty} \omega_{t, K, x^{k}}^{\prime}(\delta) \leq \omega_{t, K, x}^{\prime}(\delta) .
$$

We can suppose that $\omega_{t, K, x}^{\prime}(\delta)<\infty$. Let $\varepsilon>0$ be arbitrary and consider a subdivision $0=t_{0}<\cdots<t_{N} \leq \xi(x)$ such that

$$
\sup _{\substack{0 \leq i<N \\ t_{i} \leq s_{1}, s_{2}<t_{i+1}}} d\left(x_{s_{1}}, x_{s_{2}}\right) \leq \omega_{t, K, x}^{\prime}(\delta)+\varepsilon
$$

$t_{i+1}>t_{i}+\delta$ and $\left(t_{N}, x_{t_{N}}\right) \notin[0, t] \times K$. If $t_{N}=\xi(x)$ and $\left\{x_{s}\right\}_{s<\xi(x)} \notin S$, then we can find $\widetilde{t}_{N}$ such that $t_{N-1}+\delta<\widetilde{t}_{N}<\xi(x)$ and $x_{\widetilde{t}_{N}} \notin K$. We can suppose, possibly by replacing $t_{N}$ by $\widetilde{t}_{N}$, that

$$
t_{N}=\xi(x) \text { implies }\left\{x_{s}\right\}_{s<\xi(x)} \Subset S .
$$

Hence, for $k$ large enough, $\lambda_{t_{N}}^{k} \leq \xi\left(x^{k}\right)$ and

$$
\sup _{s<t_{N}} d\left(x_{s}, x_{\lambda_{s}^{k}}^{k}\right) \longrightarrow 0, \quad x_{\lambda_{t_{N}}^{k}}^{k} \longrightarrow x_{t_{N}}, \quad\left\|\lambda^{k}-\mathrm{id}\right\|_{t_{N}} \longrightarrow 0, \quad \text { as } k \rightarrow \infty .
$$


We deduce that, for $k$ large enough, we have $0=\lambda_{t_{0}}^{k}<\cdots<\lambda_{t_{N}}^{k} \leq \xi\left(x^{k}\right)$, $\lambda_{t_{i+1}}^{k}>\lambda_{t_{i}}^{k}+\delta,\left(\lambda_{t_{N}}^{k}, x_{\lambda_{t_{N}}^{k}}^{k}\right) \notin[0, t] \times K$, and moreover,

$$
\begin{aligned}
\sup _{\substack{0 \leq i<N \\
\lambda_{t_{i}}^{k} \leq s_{1}, s_{2}<\lambda_{t_{i+1}}^{k}}} d\left(x_{s_{1}}^{k}, x_{s_{2}}^{k}\right) \leq & \sup _{\substack{0 \leq i<N \\
t_{i} \leq s_{1}, s_{2}<t_{i+1}}} d\left(x_{s_{1}}, x_{s_{2}}\right)+2 \sup _{s<t_{N}} d\left(x_{s}, x_{\lambda_{s}^{k}}^{k}\right) \\
\leq & \omega_{t, K, x}^{\prime}(\delta)+\varepsilon+2 \sup _{s<t_{N}} d\left(x_{s}, x_{\lambda_{s}^{k}}^{k}\right) \underset{k \rightarrow \infty}{\longrightarrow} \omega_{t, K, x}^{\prime}(\delta)+\varepsilon .
\end{aligned}
$$

Therefore,

$$
\limsup _{k \rightarrow \infty} \omega_{t, K, x^{k}}^{\prime}(\delta) \leq \omega_{t, K, x}^{\prime}(\delta)+\varepsilon,
$$

and we conclude by letting $\varepsilon \rightarrow 0$.

We can give now a characterisation of the relative compactness for the local Skorokhod topology:

Theorem 2.8 (Compact sets of $\mathbb{D}_{\text {loc }}(S)$ ). For any subset $D \subset \mathbb{D}_{\text {loc }}(S), D$ is relatively compact if and only if

$$
\forall t \geq 0, K \subset S \text { compact, } \sup _{x \in D} \omega_{t, K, x}^{\prime}(\delta) \underset{\delta \rightarrow 0}{\longrightarrow} 0 .
$$

The proof follows the strategy developed in $§ 12$ pp. 121-137 from Billingsley (1999) and it is postponed to Section 4.

We conclude this section with a version of the Aldous criterion of tightness:

Proposition 2.9 (Aldous criterion). Let $\mathcal{P}$ be a subset of $\mathcal{P}\left(\mathbb{D}_{\text {loc }}(S)\right)$. If for all $t \geq 0, \varepsilon>0$, and an open subset $U \Subset S$, we have:

$$
\inf _{F \subset \mathcal{P}} \sup _{\mathbf{P} \in \mathcal{P} \backslash F} \sup _{\substack{\tau_{1} \leq \tau_{2} \\ \tau_{2} \leq\left(\tau_{1}+\delta\right) \wedge t \wedge \tau^{U}}} \mathbf{P}\left(\tau_{1}<\tau_{2}=\xi \text { or } d\left(X_{\tau_{1}}, X_{\tau_{2}}\right) \mathbb{1}_{\left\{\tau_{1}<\xi\right\}} \geq \varepsilon\right) \underset{\delta \rightarrow 0}{\longrightarrow} 0,
$$

then $\mathcal{P}$ is tight. Here the infimum is taken on all finite subsets $F \subset \mathcal{P}$ and the supremum is taken on all stopping times $\tau_{i}$.

Remark 2.10. As in Billingsley (1999), Theorem 16.9, p. 177, an equivalent condition for tightness can be obtained by replacing (2.6) by

$\inf _{F \subset \mathcal{P}} \sup _{\mathbf{P} \in \mathcal{P} \backslash F} \sup _{\tau \leq t \wedge \tau^{U}} \mathbf{P}\left(\tau<(\tau+\delta) \wedge \tau^{U}=\xi\right.$ or $\left.d\left(X_{\tau}, X_{(\tau+\delta) \wedge \tau^{U}}\right) \mathbb{1}_{\{\tau<\xi\}} \geq \varepsilon\right) \underset{\delta \rightarrow 0}{\longrightarrow} 0$,

by taking infimum on all finite subsets $F \subset \mathcal{P}$ and the supremum on all stopping times $\tau$.

In fact we will state and prove a version of the of the Aldous criterion, which is a necessary and sufficient condition, similarly as in Rebolledo (1979):

Theorem 2.11 (Tightness for $\mathbb{D}_{\text {loc }}(S)$ ). For any subset $\mathcal{P} \subset \mathcal{P}\left(\mathbb{D}_{\text {loc }}(S)\right)$ ), the following assertions are equivalent:

(1) $\mathcal{P}$ is tight,

(2) for all $t \geq 0, \varepsilon>0$ and $K$ a compact set we have

$$
\sup _{\mathbf{P} \in \mathcal{P}} \mathbf{P}\left(\omega_{t, K, X}^{\prime}(\delta) \geq \varepsilon\right) \underset{\delta \rightarrow 0}{\longrightarrow} 0,
$$


(3) for all $t \geq 0, \varepsilon>0$, and open subset $U \Subset S$ we have:

$$
\alpha(\varepsilon, t, U, \delta):=\sup _{\mathbf{P} \in \mathcal{P}} \sup _{\substack{\tau_{1} \leq \tau_{2} \leq \tau_{3} \\ \tau_{3} \leq\left(\tau_{1}+\delta\right) \wedge t \wedge \tau^{U}}} \mathbf{P}(R \geq \varepsilon) \underset{\delta \rightarrow 0}{\longrightarrow} 0,
$$

where the supremum is taken on $\tau_{i}$ stopping times and with

$$
R:= \begin{cases}d\left(X_{\tau_{1}}, X_{\tau_{2}}\right) \wedge d\left(X_{\tau_{2}}, X_{\tau_{3}}\right) & \text { if } 0<\tau_{1}<\tau_{2}<\tau_{3}<\xi, \\ d\left(X_{\tau_{2}-}, X_{\tau_{2}}\right) \wedge d\left(X_{\tau_{2}}, X_{\tau_{3}}\right) & \text { if } 0<\tau_{1}=\tau_{2}<\tau_{3}<\xi, \\ d\left(X_{\tau_{1}}, X_{\tau_{2}}\right) & \text { if } 0=\tau_{1} \leq \tau_{2}<\xi \text { or } 0<\tau_{1}<\tau_{2}<\tau_{3}=\xi, \\ d\left(X_{\tau_{2}-}, X_{\tau_{2}}\right) & \text { if } 0<\tau_{1}=\tau_{2}<\tau_{3}=\xi, \\ 0 & \text { if } \tau_{1}=\xi \text { or } 0<\tau_{1} \leq \tau_{2}=\tau_{3}, \\ \infty & \text { if } 0=\tau_{1}<\tau_{2}=\xi .\end{cases}
$$

Remark 2.12. 1) If $d$ is obtained from a metric on $S^{\Delta}$, then if $\varepsilon<d(\Delta, U)$ the expression of $\alpha(\varepsilon, t, U, \delta)$ may be simplified as follows:

$$
\alpha(\varepsilon, t, U, \delta)=\sup _{\mathbf{P} \in \mathcal{P}} \sup _{\substack{\tau_{1} \leq \tau_{2} \leq \tau_{3} \\ \tau_{3} \leq\left(\tau_{1}+\delta\right) \wedge t \wedge \tau^{U}}} \mathbf{P}(\widetilde{R} \geq \varepsilon) \underset{\delta \rightarrow 0}{\longrightarrow} 0,
$$

where the supremum is taken on $\tau_{i}$ stopping times and with

$$
\widetilde{R}:= \begin{cases}d\left(X_{\tau_{1}}, X_{\tau_{2}}\right) \wedge d\left(X_{\tau_{2}}, X_{\tau_{3}}\right) & \text { if } 0<\tau_{1}<\tau_{2}, \\ d\left(X_{\tau_{2}-}, X_{\tau_{2}}\right) \wedge d\left(X_{\tau_{2}}, X_{\tau_{3}}\right) & \text { if } 0<\tau_{1}=\tau_{2}, \\ d\left(X_{\tau_{1}}, X_{\tau_{2}}\right) & \text { if } 0=\tau_{1} .\end{cases}
$$

2) It is straightforward to verify that a subset $D \subset \mathbb{D}(S)$ is relatively compact for $\mathbb{D}(S)$ if and only if $D$ is relatively compact for $\mathbb{D}_{\text {loc }}(S)$ and

$$
\forall t \geq 0, \quad\left\{x_{s} \mid x \in D, s \leq t\right\} \Subset S .
$$

Hence we may recover the classical characterisation of compact sets of $\mathbb{D}(S)$ and the classical Aldous criterion. Moreover, we may obtain a version of Theorem 2.11 for $\mathbb{D}(S)$.

3) The difficult part of Theorem 2.11 is the implication $3 \Rightarrow 2$, and its proof is adapted from the proof of Theorem 16.10 pp. 178-179 from Billingsley (1999). Roughly speaking the assertion 3 uses

$$
\omega_{x}^{\prime \prime}(\delta):=\sup _{s_{1} \leq s_{2} \leq s_{3} \leq s_{1}+\delta} d\left(x_{s_{1}}, x_{s_{2}}\right) \wedge d\left(x_{s_{2}}, x_{s_{3}}\right),
$$

while the Aldous criterion uses

$$
\omega_{x}(\delta):=\sup _{s_{1} \leq s_{2} \leq s_{1}+\delta} d\left(x_{s_{1}}, x_{s_{2}}\right) .
$$

The term $d\left(X_{\tau_{2}-}, X_{\tau_{2}}\right)$ appears because, in contrary to the deterministic case, some stopping time may not be approximate by the left. We refer to the proof of Theorem 12.4 pp. 132-133 from Billingsley (1999) for the relation between $\omega^{\prime \prime}$ and $\omega^{\prime}$.

Proof of Theorem 2.11:

$2 \Rightarrow 1$ Consider $\left(t_{n}\right)_{n \geq 1}$ a sequence of times tending to infinity and $\left(K_{n}\right)_{n \geq 1}$ an increasing sequence of compact subsets of $S$ such that $S=\bigcup_{n} K_{n}$. Let $\eta>0$ be a real number, and for $n \geq 1$ define $\delta_{n}$ such that

$$
\sup _{\mathbf{P} \in \mathcal{P}} \mathbf{P}\left(\omega_{t_{n}, K_{n}, X}^{\prime}\left(\delta_{n}\right) \geq n^{-1}\right) \leq 2^{-n} \eta \text {. }
$$


Set

$$
D:=\left\{\forall n \in \mathbb{N}^{*}, \omega_{t_{n}, K_{n}, X}^{\prime}\left(\delta_{n}\right)<n^{-1}\right\} .
$$

By Theorem 2.8, $D$ is relatively compact and moreover

$$
\sup _{\mathbf{P} \in \mathcal{P}} \mathbf{P}\left(D^{c}\right) \leq \sum_{n \geq 1} 2^{-n} \eta=\eta
$$

so, $\mathcal{P}$ is tight.

$1 \Rightarrow 3$ Consider $\varepsilon, \eta$ two arbitrary positive real numbers. There exists a compact set $D \subset \mathbb{D}_{\text {loc }}(S)$ such that

$$
\sup _{\mathbf{P} \in \mathcal{P}} \mathbf{P}\left(D^{c}\right) \leq \eta
$$

By Theorem 2.8, there exists $\delta>0$ such that

$$
D \subset\left\{\omega_{t, \bar{U}, X}^{\prime}(\delta)<\varepsilon\right\}
$$

Since for all $\tau_{1} \leq \tau_{2} \leq \tau_{3} \leq\left(\tau_{1}+\delta\right) \wedge t \wedge \tau^{U}$ we have

$$
\left\{\omega_{t, \bar{U}, X}^{\prime}(\delta)<\varepsilon\right\} \subset\{R<\varepsilon\},
$$

we conclude that

$$
\sup _{\mathbf{P} \in \mathcal{P}} \sup _{\substack{\tau_{1} \leq \tau_{2} \leq \tau_{3} \\ \tau_{3} \leq\left(\tau_{1}+\delta\right) \wedge t \wedge \tau^{U}}} \mathbf{P}(R \geq \varepsilon) \leq \eta .
$$

$3 \Rightarrow 2$ For all $\varepsilon>0, t \geq 0$ and open subset $U \Subset S$, up to consider $\widetilde{\tau}_{i}:=\tau_{i} \wedge\left(\tau_{1}+\right.$ $\delta) \wedge t \wedge \tau^{U}$ we have a new expression of $\alpha(\varepsilon, t, U, \delta)$ :

$$
\alpha(\varepsilon, t, U, \delta)=\sup _{\mathbf{P} \in \mathcal{P}} \sup _{\tau_{1} \leq \tau_{2} \leq \tau_{3} \leq \xi} \mathbf{P}\left(R \geq \varepsilon, \tau_{3} \leq\left(\tau_{1}+\delta\right) \wedge t \wedge \tau^{U}\right) \underset{\delta \rightarrow 0}{\longrightarrow} 0 .
$$

Consider $\varepsilon_{0}>0, t \geq 0$ and $K$ a compact subset of $S$. We need to prove that

$$
\inf _{\mathbf{P} \in \mathcal{P}} \mathbf{P}\left(\omega_{t, K, X}^{\prime}(\delta)<\varepsilon_{0}\right) \underset{\delta \rightarrow 0}{\longrightarrow} 1 .
$$

Choose $0<\varepsilon_{1}<\varepsilon_{0} / 4$ such that

$$
U:=\left\{y \in S \mid d(y, K)<\varepsilon_{1}\right\} \Subset S .
$$

For $n \in \mathbb{N}$ and $\varepsilon>0$, define inductively the stopping times (see Corollary 2.3)

$$
\begin{aligned}
& \tau_{0}:=0, \\
& \tau_{n}^{\varepsilon}:=\inf \left\{s>\tau_{n} \mid d\left(X_{\tau_{n}}, X_{s}\right) \vee d\left(X_{\tau_{n}}, X_{s-}\right) \geq \varepsilon\right\} \wedge(t+2) \wedge \tau^{U}, \\
& \tau_{n+1}:=\tau_{n}^{\varepsilon_{1}},
\end{aligned}
$$

It is clear that $\tau_{n}^{\varepsilon}$ increases to $\tau_{n+1}$ when $\varepsilon$ increases to $\varepsilon_{1}$. If we choose $0<\varepsilon_{2}<\varepsilon_{1}$, then for all $\mathbf{P} \in \mathcal{P}$,

$$
\begin{aligned}
& \limsup _{\substack{\varepsilon \rightarrow \varepsilon_{1} \\
\varepsilon<\varepsilon_{1}}} \mathbf{P}\left(X_{\tau_{n}} \in K, \tau_{n}^{\varepsilon}<\xi, d\left(X_{\tau_{n}}, X_{\tau_{n}^{\varepsilon}}\right) \leq \varepsilon_{2}, \tau_{n}^{\varepsilon} \leq t+1\right) \\
& \quad \leq \mathbf{P}\left(\limsup _{\substack{\varepsilon \rightarrow \varepsilon_{1} \\
\varepsilon<\varepsilon_{1}}}\left\{X_{\tau_{n}} \in K, \tau_{n}^{\varepsilon}<\xi, d\left(X_{\tau_{n}}, X_{\tau_{n}^{\varepsilon}}\right) \leq \varepsilon_{2}, \tau_{n}^{\varepsilon} \leq t+1\right\}\right)=\mathbf{P}(\emptyset)=0 .
\end{aligned}
$$


For all $\mathbf{P} \in \mathcal{P}, \delta \leq 1$ and $0<\varepsilon<\varepsilon_{1}$ we have using the expression (2.7) with stopping times $0 \leq \tau_{0}^{\varepsilon} \leq \tau_{0}^{\varepsilon_{1}}=\tau_{1}$

$$
\begin{aligned}
\mathbf{P}\left(X_{0} \in K, \tau_{0}^{\varepsilon} \leq \delta\right)= & \mathbf{P}\left(X_{0} \in K, X_{\tau_{0}^{\varepsilon}} \notin B\left(X_{0}, \varepsilon_{2}\right), \tau_{0}^{\varepsilon} \leq \delta\right) \\
& +\mathbf{P}\left(X_{0} \in K, \tau_{0}^{\varepsilon}<\xi, d\left(X_{0}, X_{\tau_{0}^{\varepsilon}}\right)<\varepsilon_{2}, \tau_{0}^{\varepsilon} \leq \delta\right) \\
\leq & \alpha\left(\varepsilon_{2}, t+2, U, \delta\right) \\
& +\mathbf{P}\left(X_{0} \in K, \tau_{0}^{\varepsilon}<\xi, d\left(X_{0}, X_{\tau_{0}^{\varepsilon}}\right)<\varepsilon_{2}, \tau_{0}^{\varepsilon} \leq t+1\right)
\end{aligned}
$$

so, letting $\varepsilon \rightarrow \varepsilon_{1}$, since $\tau_{0}^{\varepsilon} \uparrow \tau_{1}$, by (2.8) we obtain

$$
\mathbf{P}\left(X_{0} \in K, \tau_{1} \leq \delta\right) \leq \alpha\left(\varepsilon_{2}, t+2, U, \delta\right) .
$$

For all $\mathbf{P} \in \mathcal{P}, \delta \leq 1, n \in \mathbb{N}$ and $0<\varepsilon<\varepsilon_{1}$ we have also using the expression (2.7) with stopping times $\tau_{n} \leq \tau_{n}^{\varepsilon} \leq \tau_{n+1}$ and $\tau_{n} \leq \tau_{n}^{\varepsilon} \leq \tau_{n+1}^{\varepsilon}$

$$
\begin{aligned}
& \mathbf{P}\left(\tau_{n+1} \leq t, X_{\tau_{n}}, X_{\tau_{n+1}} \in K, \tau_{n+1}^{\varepsilon}-\tau_{n} \leq \delta\right) \\
& \leq \mathbf{P}\left(X_{\tau_{n}} \in K, \tau_{n}^{\varepsilon}<\xi, d\left(X_{\tau_{n}}, X_{\tau_{n}^{\varepsilon}}\right)<\varepsilon_{2}, \tau_{n}^{\varepsilon} \leq t+1\right) \\
& \quad+\mathbf{P}\left(X_{\tau_{n+1}} \in K, \tau_{n+1}^{\varepsilon}<\xi, d\left(X_{\tau_{n+1}}, X_{\tau_{n+1}^{\varepsilon}}\right)<\varepsilon_{2}, \tau_{n+1}^{\varepsilon} \leq t+1\right) \\
& \quad+\mathbf{P}\left(\tau_{n+1} \leq t, X_{\tau_{n}}, X_{\tau_{n+1}} \in K, d\left(X_{\tau_{n}}, X_{\tau_{n}^{\varepsilon}}\right) \geq \varepsilon_{2},\right. \\
& \left.\quad d\left(X_{\tau_{n}^{\varepsilon}}, X_{\tau_{n+1}}\right) \geq \frac{\varepsilon_{2}}{2}, \tau_{n+1}-\tau_{n} \leq \delta\right) \\
& \quad+\mathbf{P}\left(\tau_{n+1} \leq t, X_{\tau_{n}}, X_{\tau_{n+1}} \in K, d\left(X_{\tau_{n}}, X_{\tau_{n}^{\varepsilon}}\right) \geq \varepsilon_{2}, X_{\tau_{n+1}^{\varepsilon}} \notin B\left(X_{\tau_{n}^{\varepsilon}}, \frac{\varepsilon_{2}}{2}\right),\right. \\
& \left.\quad \tau_{n+1}^{\varepsilon}-\tau_{n} \leq \delta\right) \\
& \leq \mathbf{P}\left(X_{\tau_{n}} \in K, \tau_{n}^{\varepsilon}<\xi, d\left(X_{\tau_{n}}, X_{\tau_{n}^{\varepsilon}}\right) \leq \varepsilon_{2}, \tau_{n}^{\varepsilon} \leq t+1\right) \\
& \quad+\mathbf{P}\left(X_{\tau_{n+1}} \in K, \tau_{n+1}^{\varepsilon}<\xi, d\left(X_{\tau_{n+1}}, X_{\tau_{n+1}^{\varepsilon}}\right) \leq \varepsilon_{2}, \tau_{n+1}^{\varepsilon} \leq t+1\right) \\
& \quad+2 \alpha\left(\frac{\varepsilon_{2}}{2}, t+2, U, \delta\right)
\end{aligned}
$$

so, letting $\varepsilon \rightarrow \varepsilon_{1}$, since $\tau_{n+1}^{\varepsilon} \uparrow \tau_{n+2}$, by (2.8) we obtain

$$
\mathbf{P}\left(\tau_{n+1} \leq t, X_{\tau_{n}}, X_{\tau_{n+1}} \in K, \tau_{n+2}-\tau_{n} \leq \delta\right) \leq 2 \alpha\left(\frac{\varepsilon_{2}}{2}, t+2, U, \delta\right) .
$$

For all $\mathbf{P} \in \mathcal{P}, \delta \leq 1, n \in \mathbb{N}^{*}$ and $0<\varepsilon<\varepsilon_{1}$ we can write using the expression (2.7) with stopping times $\tau_{n} \leq \tau_{n} \leq \tau_{n}^{\varepsilon}$

$$
\begin{aligned}
\mathbf{P}\left(\tau_{n}\right. & \left.\leq t, X_{\tau_{n}} \in K, d\left(X_{\tau_{n}-}, X_{\tau_{n}}\right) \geq \varepsilon_{2}, \tau_{n}^{\varepsilon}-\tau_{n} \leq \delta\right) \\
& \leq \mathbf{P}\left(X_{\tau_{n}} \in K, \tau_{n}^{\varepsilon}<\xi, d\left(X_{\tau_{n}}, X_{\tau_{n}^{\varepsilon}}\right)<\varepsilon_{2}, \tau_{n}^{\varepsilon} \leq t+1\right) \\
& +\mathbf{P}\left(\tau_{n} \leq t, X_{\tau_{n}} \in K, d\left(X_{\tau_{n}-}, X_{\tau_{n}}\right) \geq \varepsilon_{2}, X_{\tau_{n}^{\varepsilon}} \notin B\left(X_{\tau_{n}}, \varepsilon_{2}\right), \tau_{n}^{\varepsilon}-\tau_{n} \leq \delta\right) \\
& \leq \mathbf{P}\left(X_{\tau_{n}} \in K, \tau_{n}^{\varepsilon}<\xi, d\left(X_{\tau_{n}}, X_{\tau_{n}^{\varepsilon}}\right) \leq \varepsilon_{2}, \tau_{n}^{\varepsilon} \leq t+1\right)+\alpha\left(\varepsilon_{2}, t+2, U, \delta\right)
\end{aligned}
$$

so, letting $\varepsilon \rightarrow \varepsilon_{1}$, since $\tau_{n}^{\varepsilon \uparrow} \tau_{n+1}$, by (2.8) we obtain

$$
\mathbf{P}\left(\tau_{n} \leq t, X_{\tau_{n}} \in K, d\left(X_{\tau_{n}-}, X_{\tau_{n}}\right) \geq \varepsilon_{2}, \tau_{n+1}-\tau_{n} \leq \delta\right) \leq \alpha\left(\varepsilon_{2}, t+2, U, \delta\right) .
$$

Let $m \in 2 \mathbb{N}$ and $0<\delta^{\prime} \leq 1$ be such that $m>2 t / \delta^{\prime}$ and denote the event

$$
A:=\left\{\tau_{m} \leq t \text { and } \forall n \leq m, X_{\tau_{n}} \in K\right\} \text {. }
$$


Then for all $0 \leq i<m$, thanks to $(2.10)$

$$
\mathbf{E}\left[\tau_{i+2}-\tau_{i} \mid A\right] \geq \delta^{\prime} \mathbf{P}\left(\tau_{i+2}-\tau_{i} \geq \delta^{\prime} \mid A\right) \geq \delta^{\prime}\left(1-\frac{2 \alpha\left(\frac{\varepsilon_{2}}{2}, t+2, U, \delta^{\prime}\right)}{\mathbf{P}(A)}\right) .
$$

Hence

$$
t \geq \mathbf{E}\left[\tau_{m} \mid A\right]=\sum_{i=0}^{(m-2) / 2} \mathbf{E}\left[\tau_{2 i+2}-\tau_{2 i} \mid A\right] \geq \frac{m \delta^{\prime}}{2}\left(1-\frac{2 \alpha\left(\frac{\varepsilon_{2}}{2}, t+2, U, \delta^{\prime}\right)}{\mathbf{P}(A)}\right)
$$

so,

$$
\mathbf{P}(A) \leq \frac{2 \alpha\left(\frac{\varepsilon_{2}}{2}, t+2, U, \delta^{\prime}\right)}{1-2 t /\left(m \delta^{\prime}\right)} .
$$

Taking $0<\delta \leq 1$ and setting

$$
B_{m, \delta}:=\left\{\begin{array}{l}
\left(\tau_{m}, X_{\tau_{0}}, \ldots, X_{\tau_{m}}\right) \notin[0, t] \times K^{m+1}, \\
X_{0} \in K \Rightarrow \tau_{1}>\delta \\
\forall 0 \leq n \leq m-2, \tau_{n+1} \leq t \text { and } X_{\tau_{n}}, X_{\tau_{n+1}} \in K \Rightarrow \tau_{n+2}-\tau_{n}>\delta, \\
\forall 0 \leq n<m, \tau_{n} \leq t, X_{\tau_{n}} \in K, d\left(X_{\tau_{n}-}, X_{\tau_{n}}\right) \geq \varepsilon_{2} \Rightarrow \tau_{n+1}-\tau_{n}>\delta
\end{array}\right\},
$$

by (2.9), (2.10), (2.11) and (2.12) we obtain that

$$
\begin{aligned}
\inf _{\mathbf{P} \in \mathcal{P}} \mathbf{P}\left(B_{m, \delta}\right) \geq & 1-\frac{2 \alpha\left(\frac{\varepsilon_{2}}{2}, t+2, U, \delta^{\prime}\right)}{1-2 t /\left(m \delta^{\prime}\right)}-\alpha\left(\varepsilon_{2}, t+2, U, \delta\right) \\
& -2(m-1) \alpha\left(\frac{\varepsilon_{2}}{2}, t+2, U, \delta\right)-m \alpha\left(\varepsilon_{2}, t+2, U, \delta\right) .
\end{aligned}
$$

Hence

$$
\sup _{m \in \mathbb{N}} \inf _{\mathbf{P} \in \mathcal{P}} \mathbf{P}\left(B_{m, \delta}\right) \underset{\delta \rightarrow 0}{\longrightarrow} 1 .
$$

Recalling that $\varepsilon_{1}<4 \varepsilon_{0}$, a straightforward computation gives

$$
B_{m, \delta} \subset\left\{\omega_{t, K, X}^{\prime}(\delta)<\varepsilon_{0}\right\} .
$$

We conclude that

$$
\inf _{\mathbf{P} \in \mathcal{P}} \mathbf{P}\left(\omega_{t, K, X}^{\prime}(\delta)<\varepsilon_{0}\right) \underset{\delta \rightarrow 0}{\longrightarrow} 1
$$

\section{Time change and Skorokhod topologies}

3.1. Definition and properties of time change. First we give the definition of the time change mapping (see also §6.1 pp. 306-311 from Ethier and Kurtz, 1986, §V.26 pp. 175-177 from Rogers and Williams, 2000).

Definition 3.1 (Time Change). Let us introduce $\mathrm{C}^{\neq 0}\left(S, \mathbb{R}_{+}\right):=\left\{g: S \rightarrow \mathbb{R}_{+} \mid\{g=0\}\right.$ is closed and $g$ is continuous on $\left.\{g \neq 0\}\right\}$, and for $g \in \mathrm{C}^{\neq 0}\left(S, \mathbb{R}_{+}\right), x \in \mathbb{D}_{\exp }(S)$ and $t \in[0, \infty]$ we denote

$\tau_{t}^{g}(x):=\inf \left\{s \geq 0 \mid A_{s}^{g}(x) \geq t\right\}$, where $A_{t}^{g}(x):= \begin{cases}\int_{0}^{t} \frac{\mathrm{d} u}{g\left(x_{u}\right)}, & \text { if } t \in\left[0, \tau^{\{g \neq 0\}}(x)\right], \\ \infty & \text { otherwise. }\end{cases}$ 
For $g \in \mathrm{C}^{\neq 0}\left(S, \mathbb{R}_{+}\right)$, we define a time change mapping, which is $\mathcal{F}$-measurable,

$$
\begin{aligned}
& g \cdot X: \mathbb{D}_{\exp }(S) \rightarrow \mathbb{D}_{\exp }(S) \\
& x \quad \mapsto \quad g \cdot x,
\end{aligned}
$$

as follows: for $t \in \mathbb{R}_{+}$

$$
(g \cdot X)_{t}:= \begin{cases}X_{\tau_{\infty}^{g}-} & \text { if } t \geq A_{\tau_{\infty}^{g}}^{g}, X_{\tau_{\infty}^{g}-} \text { exists and belongs to }\{g=0\}, \\ X_{\tau_{t}^{g}} & \text { otherwise. }\end{cases}
$$

For $g \in \mathrm{C}^{\neq 0}\left(S, \mathbb{R}_{+}\right)$and $\mathbf{P} \in \mathcal{P}\left(\mathbb{D}_{\exp }(S)\right)$, we also define $g \cdot \mathbf{P}$ the pushforward of $\mathbf{P}$ by $x \mapsto g \cdot x$.

The fact that this mapping is measurable will be proved in the next section.

Remark 3.2. Let us stress that, by using Corollary $2.3, \tau_{t}^{g}$ is a stopping time. In particular, the following stopping time will play a crucial role:

$$
\tau_{\infty}^{g}=\tau^{\{g \neq 0\}}:=\xi \wedge \inf \left\{t \geq 0 \mid g\left(X_{t-}\right) \wedge g\left(X_{t}\right)=0\right\} .
$$

The time of explosion of $g \cdot X$ is given by

$$
\xi(g \cdot X)= \begin{cases}\infty & \text { if } \tau_{\infty}^{g}<\xi \text { or } X_{\xi-} \text { exists and belongs to }\{g=0\} \\ A_{\xi}^{g} & \text { otherwise. }\end{cases}
$$

Roughly speaking, $g \cdot X$ is given by $(g \cdot X)_{t}:=X_{\tau_{t}^{g}}$ where $t \mapsto \tau_{t}^{g}$ is the solution of $\dot{\tau}_{t}^{g}=g\left(X_{\tau_{t}^{g}}\right)$, on the time interval $\left[0, \tau_{\infty}^{g}\right)$.

\section{Proposition 3.3.}

(1) For $U \subset S$ an open subset, by identifying

$$
\mathrm{C}\left(U, \mathbb{R}_{+}\right)=\left\{g \in \mathrm{C}^{\neq 0}\left(S, \mathbb{R}_{+}\right) \mid\{g \neq 0\} \subset U \text { and } g \text { is continuous on } U\right\} \text {, }
$$

the time change mapping

$$
\begin{aligned}
& \mathrm{C}\left(U, \mathbb{R}_{+}\right) \times \mathbb{D}_{\exp }(S) \quad \rightarrow \quad \mathbb{D}_{\exp }(S) \\
& (g, x) \quad \mapsto \quad g \cdot x,
\end{aligned}
$$

is measurable between $\mathcal{B}\left(\mathrm{C}\left(U, \mathbb{R}_{+}\right)\right) \otimes \mathcal{F}$ and $\mathcal{F}$.

(2) If $g_{1}, g_{2} \in \mathrm{C}^{\neq 0}\left(S, \mathbb{R}_{+}\right)$and $x \in \mathbb{D}_{\text {exp }}(S)$, then $g_{1} \cdot\left(g_{2} \cdot x\right)=\left(g_{1} g_{2}\right) \cdot x$.

(3) If $g$ is bounded and belongs to $\mathrm{C}^{\neq 0}\left(S, \mathbb{R}_{+}\right)$, and $x \in \mathbb{D}(S)$, then $g \cdot x \in \mathbb{D}(S)$.

(4) Define

$$
\begin{aligned}
& \widetilde{\mathrm{C}}^{\neq 0}\left(S, \mathbb{R}_{+}\right):=\left\{g \in \mathrm{C}^{\neq 0}\left(S, \mathbb{R}_{+}\right) \mid \forall K \subset S \text { compact, } g(K) \text { is bounded }\right\} \text {. } \\
& \text { If } g \in \widetilde{\mathrm{C}}^{\neq 0}\left(S, \mathbb{R}_{+}\right) \text {and } x \in \mathbb{D}_{\text {loc }}(S) \text {, then } g \cdot x \in \mathbb{D}_{\text {loc }}(S) \text {. }
\end{aligned}
$$

Proof: The first point is straightforward by using Proposition 2.2, while the second point is a direct consequence of the time change definition and, in particular, of the first part of (3.2). The third point can be easily deduced because,

$$
\xi(g \cdot x) \geq \int_{0}^{\infty} \frac{\mathrm{d} s}{g\left(x_{s}\right)} \geq \int_{0}^{\infty} \frac{\mathrm{d} s}{\|g\|}=\infty .
$$

To prove the fourth point we suppose that $\xi(g \cdot x)<\infty$ and $\left\{g \cdot x_{s}\right\}_{s<\xi(g \cdot x)} \Subset S$. Then, $\left\{x_{s}\right\}_{s<\xi(x)}=\left\{g \cdot x_{s}\right\}_{s<\xi(g \cdot x)}$ so,

$$
\infty>\xi(g \cdot x)=\int_{0}^{\xi(x)} \frac{\mathrm{d} s}{g\left(x_{s}\right)} \geq \frac{\xi(x)}{\|g\|_{\left\{x_{s}\right\}_{s<\xi(x)}}} .
$$

Hence $\xi(x)<\infty$ and so, $g \cdot x_{\xi(g \cdot x)-}=x_{\xi(x)-}$ exists. 
Remark 3.4. It can be proved that if $g \in \mathrm{C}^{\neq 0}\left(S, \mathbb{R}_{+}\right)$and $\left(\mathbf{P}_{a}\right)_{a \in S}$ is a strong Markov family, then $\left(g \cdot \mathbf{P}_{a}\right)_{a \in S}$ is a Markov family. Furthermore, if $\left(\mathbf{P}_{a}\right)_{a \in S}$ is a $\mathcal{F}_{t+}$-strong Markov family, then $\left(g \cdot \mathbf{P}_{a}\right)_{a \in S}$ is a $\mathcal{F}_{t+}$-strong Markov family. We will not use these properties here hence we skip the proofs of these statements.

Another interesting fact is the following:

Theorem 3.5 (Continuity of the time change). For couples $(g, x) \in \widetilde{\mathrm{C}}^{\neq 0}\left(S, \mathbb{R}_{+}\right) \times$ $\mathbb{D}_{\text {loc }}(S)$ consider the following two conditions:

$$
\tau_{\infty}^{g}(x)<\xi(x) \text { implies } \int_{0}^{\tau_{\infty}^{g}(x)+} \frac{\mathrm{d} s}{g\left(x_{s}\right)}=\infty,
$$

and

$$
A_{\tau_{\infty}^{g}(x)}^{g}(x)<\infty, x_{\tau_{\infty}^{g}(x)-} \text { exists in } S \text { and } g\left(x_{\tau_{\infty}^{g}(x)-}\right)=0 \text { imply } x_{\tau_{\infty}^{g}(x)-}=x_{\tau_{\infty}^{g}(x)} .
$$

Introduce the set

$$
B_{\mathrm{tc}}:=\left\{(g, x) \in \widetilde{\mathrm{C}}^{\neq 0}\left(S, \mathbb{R}_{+}\right) \times \mathbb{D}_{\text {loc }}(S) \mid \text { conditions (3.4) and (3.5) hold }\right\} .
$$

Then the time change

$$
\begin{array}{ccc}
\widetilde{\mathrm{C}}^{\neq 0}\left(S, \mathbb{R}_{+}\right) \times \mathbb{D}_{l o c}(S) & \rightarrow & \mathbb{D}_{l o c}(S) \\
(g, x) & \mapsto & g \cdot x
\end{array}
$$

is continuous on $B_{\mathrm{tc}}$ when we endow respectively $\widetilde{\mathrm{C}}^{\neq 0}\left(S, \mathbb{R}_{+}\right)$with the topology of uniform convergence on compact sets and $\mathbb{D}_{\text {loc }}(S)$ with the local Skorokhod topology. In particular

$$
\begin{array}{ccc}
\mathrm{C}\left(S, \mathbb{R}_{+}^{*}\right) \times \mathbb{D}_{l o c}(S) & \rightarrow & \mathbb{D}_{l o c}(S) \\
(g, x) & \mapsto & g \cdot x
\end{array}
$$

is continuous for the topologies of uniform convergence on compact sets and local Skorokhod topology. Here and elsewhere we denote by $\mathbb{R}_{+}^{*}=\mathbb{R}_{+} \backslash\{0\}$ the set of positive real numbers.

Remark 3.6. 1) It is not difficult to prove that $B_{\mathrm{tc}}$ is the continuity set.

2) If $(g, x) \in B_{\mathrm{tc}}$ and $h \in \widetilde{\mathrm{C}}^{\neq 0}\left(S, \mathbb{R}_{+}\right)$is such that $\{h=0\}=\{g=0\}$ and $h \leq C g$ for a constant $C \in \mathbb{R}_{+}$, then $(h, x) \in B_{\mathrm{tc}}$.

3) More generally, let $B_{0}$ be the set of $(g, x) \in \widetilde{\mathrm{C}}^{\neq 0}\left(S, \mathbb{R}_{+}\right) \times \mathbb{D}_{\text {loc }}(S)$ such that

$$
\begin{aligned}
& \tau_{\infty}^{g}(x)<\infty \Rightarrow \forall t \geq 0, x_{\tau_{\infty}^{g}(x)+t}=x_{\tau_{\infty}^{g}(x)}, \\
& x_{\tau_{\infty}^{g}(x)-} \text { exists in } S \text { and } g\left(x_{\tau_{\infty}^{g}(x)-}\right)=0 \Rightarrow x_{\tau_{\infty}^{g}(x)-}=x_{\tau_{\infty}^{g}(x)} .
\end{aligned}
$$

Then

$$
\left\{(g, g \cdot x) \mid(g, x) \in \widetilde{\mathrm{C}}^{\neq 0}\left(S, \mathbb{R}_{+}\right) \times \mathbb{D}_{\mathrm{loc}}(S)\right\} \subset B_{0} \subset B_{\mathrm{tc}} .
$$

To simplify the proof of the theorem we use a technical result containing a construction of a sequence of bi-Lipschitz bijections $\left(\lambda^{k}\right)_{k}$ useful when proving the convergence. Before stating this result let us note that, for any $(g, x) \in$ $\widetilde{\mathrm{C}}^{\neq 0}\left(S, \mathbb{R}_{+}\right) \times \mathbb{D}_{\text {loc }}(S)$ and any $t \leq \tau_{\infty}^{g}(x)$ such that $\left\{x_{s}\right\}_{s<t} \Subset\{g \neq 0\}$, by using (3.1), $A_{t}^{g}(x)<\infty$.

Lemma 3.7. Take a metric d of $S$. Let $x, x^{k} \in \mathbb{D}_{l o c}(S)$ and $g, g_{k} \in \widetilde{\mathrm{C}}^{\neq 0}\left(S, \mathbb{R}_{+}\right)$ be such that $\left(g_{k}, x^{k}\right)$ converges to $(g, x)$, as $k \rightarrow \infty$. Let $t \leq \tau_{\infty}^{g}(x)$ be such that $\left\{x_{s}\right\}_{s<t} \Subset\{g \neq 0\}$. Then, 
i) there exists a sequence $\left(\lambda^{k}\right)_{k} \in \widetilde{\Lambda}^{\mathbb{N}}$ such that, for $k$ large enough $\lambda_{A_{t}^{g}(x)}^{k} \leq$ $\xi\left(g_{k} \cdot x^{k}\right)$ and

$\left\|\log \dot{\lambda}^{k}\right\| \rightarrow 0, \quad \sup _{v<A_{t}^{g}(x)} d\left(g \cdot x_{v}, g_{k} \cdot x_{\lambda_{v}^{k}}^{k}\right) \rightarrow 0, \quad g_{k} \cdot x_{\lambda_{A_{t}^{k}(x)}^{k}}^{k} \rightarrow g \cdot x_{A_{t}^{g}(x)}, \quad$ as $k \rightarrow \infty$.

ii) Moreover, if $\tau_{\infty}^{g}(x)<\xi(x)$ and $\int_{0}^{\tau_{\infty}^{g}(x)+} \frac{\mathrm{d} s}{g\left(x_{s}\right)}=\infty,\left(\lambda^{k}\right)_{k}$ may be chosen such that for any $v \geq 0$ and $k$ large enough $\lambda_{A_{t}^{g}(x)+v}^{k}<\xi\left(g_{k} \cdot x^{k}\right)$ and

$$
\limsup _{k \rightarrow \infty} \sup _{A_{t}^{g}(x) \leq w \leq A_{t}^{g}(x)+v} d\left(g_{k} \cdot x_{\lambda_{w}^{k}}^{k},\left\{x_{s}\right\}_{t \leq s \leq \tau_{\infty}^{g}(x)}\right)=0 .
$$

We postpone the proof of the lemma, and we give the proof of the continuity of time change:

Proof of Theorem 3.5: We remark first that

$$
B_{\text {tc }}=B_{1} \cup B_{2} \cup B_{3} \cup B_{4},
$$

with

$$
\begin{aligned}
& B_{1}:=\left\{A_{\tau_{\infty}^{g}(x)}^{g}(x)=\infty \text { or }\left\{x_{s}\right\}_{s<\tau_{\infty}^{g}(x)} \notin S\right\}, \\
& B_{2}:=\left\{\tau_{\infty}^{g}(x)=\xi(x)<\infty \text { and }\left\{x_{s}\right\}_{s<\tau_{\infty}^{g}(x)} \Subset\{g \neq 0\}\right\}, \\
& B_{3}:=\left\{\tau_{\infty}^{g}(x)<\xi(x), x_{\tau_{\infty}^{g}(x)-}=x_{\tau_{\infty}^{g}(x)}, A_{\tau_{\infty}^{g}(x)}^{g}(x)<\infty \text { and } \int_{0}^{\tau_{\infty}^{g}(x)^{+}} \frac{\mathrm{d} s}{g\left(x_{s}\right)}=\infty\right\}, \\
& B_{4}:=\left\{\tau_{\infty}^{g}(x)<\xi(x), g\left(x_{\tau_{\infty}^{g}(x)-}\right) \neq 0 \text { and } \int_{0}^{\tau_{\infty}^{g}(x)^{+}} \frac{\mathrm{d} s}{g\left(x_{s}\right)}=\infty\right\} .
\end{aligned}
$$

Let $x, x^{k} \in \mathbb{D}_{\text {loc }}(S)$ and $g, g_{k} \in \widetilde{\mathrm{C}}^{\neq 0}\left(S, \mathbb{R}_{+}\right)$be such that $\left(g_{k}, x^{k}\right)$ converge to $(g, x)$ and $(g, x) \in B$. We need to prove that

$$
g_{k} \cdot x^{k} \underset{k \rightarrow \infty}{\stackrel{\mathbb{P}_{\mathrm{loc}}(S)}{\longrightarrow}} g \cdot x
$$

and we will decompose the proof with respect to values of $i$ such that $(g, x) \in B_{i}$.

- If $(g, x) \in B_{1}$, we use the first part of Lemma 3.7 for all $t<\tau_{\infty}^{g}(x)$. We obtain that $A_{t}^{g}(x)<\xi(g \cdot x)$. Since $A_{t}^{g}(x)$ tends to $\xi(g \cdot x)$, when $t$ tends to $\tau_{\infty}^{g}(x)$, by a diagonal extraction procedure we deduce (3.8).

- If $(g, x) \in B_{2}$, it suffices to apply the first part of Lemma 3.7 to $t:=\xi(x)$ and $A_{t}^{g}(x)=\xi(g \cdot x)$.

- If $(g, x) \in B_{3}$, let $t<\tau_{\infty}^{g}(x)$ be. Then, by the third part of Lemma 3.7 there exists $\lambda^{k} \in \widetilde{\Lambda}$ such that, for any $v \geq 0$, for $k$ large enough, $\lambda_{A_{t}^{g}(x)+v}^{k}<$ $\xi\left(g_{k} \cdot x^{k}\right)$ and

$\left\|\log \dot{\lambda}^{k}\right\| \underset{k \rightarrow \infty}{\longrightarrow} 0, \quad \limsup _{k \rightarrow \infty} \sup _{w \leq A_{t}^{g}(x)+v} d\left(g \cdot x_{w}, g_{k} \cdot x_{\lambda_{w}^{k}}^{k}\right) \leq 2 d\left(x_{\tau_{\infty}^{g}(x)},\left\{x_{s}\right\}_{t \leq s \leq \tau_{\infty}^{g}(x)}\right)$.

Since $x$ is continuous at $\tau_{\infty}^{g}(x)$, we conclude by a diagonal extraction procedure, by letting $t$ tends to $\tau_{\infty}^{g}(x)$ and $v \rightarrow \infty$. 
- If $(g, x) \in B_{4}$, let $t=\tau_{\infty}^{g}(x)$ be. By the second part of Lemma 3.7 there exists $\lambda^{k} \in \widetilde{\Lambda}$ such that, for any $v \geq 0$, for $k$ large enough $\lambda_{A_{t}^{g}(x)+v}^{k}<$ $\xi\left(g_{k} \cdot x^{k}\right)$, and

$$
\left\|\log \dot{\lambda}^{k}\right\| \underset{k \rightarrow \infty}{\longrightarrow} 0, \quad \sup _{w \leq A_{t}^{g}(x)+v} d\left(g \cdot x_{w}, g_{k} \cdot x_{\lambda_{w}^{k}}^{k}\right) \underset{k \rightarrow \infty}{\longrightarrow} 0 .
$$

We conclude by a diagonal extraction procedure and letting $v \rightarrow \infty$.

Proof of Lemma 3.\%: Let $\widetilde{\lambda}^{k} \in \widetilde{\Lambda}$ be as in Theorem 2.4 and to simplify notations define, for $s \geq 0$

$$
\begin{array}{rlrl}
\tau_{s} & :=\tau_{s}^{g}(x), & A_{s}:=A_{s}^{g}(x), \\
\tau_{s}^{k}:=\tau_{s}^{g_{k}}\left(x^{k}\right), & A_{s}^{k}:=A_{s}^{g_{k}}\left(x^{k}\right),
\end{array}
$$

and $u:=A_{t}$. Since $\tau_{u}=t \leq \xi(x)$ and $\left\{x_{s}\right\}_{s<t} \Subset S$ we have, for $k$ large enough $\widetilde{\lambda}_{t}^{k} \leq$ $\xi\left(x^{k}\right)$, and $\left\|\log \dot{\vec{\lambda}}^{k}\right\|_{t} \longrightarrow 0, \sup _{s<t} d\left(x_{s}, x_{\widetilde{\lambda}_{s}^{k}}^{k}\right) \longrightarrow 0$ and $x_{\widetilde{\lambda}_{t}^{k}}^{k} \longrightarrow x_{t}$, as $k \rightarrow \infty$. Since $\left\{x_{s}\right\}_{s<t} \Subset\{g \neq 0\}$, we deduce that for $k$ large enough $\left\{x_{s}^{k}\right\}_{s<\tilde{\lambda}_{t}^{k}} \Subset\left\{g_{k} \neq 0\right\}$. Define then $\lambda^{k} \in \widetilde{\Lambda}$ by

$$
\begin{cases}\lambda_{v}^{k}:=A_{\widetilde{\lambda}_{\tau_{v}}^{k}}^{k}=\int_{0}^{v} \frac{g\left(x_{\tau_{w}}\right)}{g_{k}\left(x_{\widetilde{\lambda}_{\tau_{w}}^{k}}^{k}\right)} \dot{\vec{\lambda}}_{\tau_{w}}^{k} \mathrm{~d} w & \text { if } v \leq u, \\ \dot{\lambda}_{v}^{k}=1 & \text { if } v>u .\end{cases}
$$

Since $\widetilde{\lambda}_{t}^{k} \leq \tau_{\infty}^{k}$ we have

$$
\lambda_{u}^{k} \leq A_{\tau_{\infty}^{k}}^{k} \leq \xi\left(g_{k} \cdot x^{k}\right)
$$

now we obtain

$$
\begin{aligned}
& \sup _{v<u} d\left(g \cdot x_{v}, g_{k} \cdot x_{\lambda_{v}^{k}}^{k}\right)=\sup _{v<u} d\left(x_{\tau_{v}}, x_{\widetilde{\lambda}_{\tau_{v}}^{k}}^{k}\right)=\sup _{s<t} d\left(x_{s}, x_{\widetilde{\lambda}_{s}^{k}}^{k}\right) \underset{k \rightarrow \infty}{\longrightarrow} 0, \\
& g_{k} \cdot x_{\lambda_{u}^{k}}^{k}=x_{\widetilde{\lambda}_{t}^{k}}^{k} \underset{k \rightarrow \infty}{\longrightarrow} x_{t}=g \cdot x_{u} \\
& \left\|\log \dot{\lambda}^{k}\right\|=\operatorname{esssup}_{v \leq u}\left|\log \frac{\dot{\tilde{\lambda}}_{\tau_{v}}^{k} g\left(x_{\tau_{v}}\right)}{g_{k}\left(x_{\widetilde{\lambda}_{\tau_{v}}^{k}}^{k}\right)}\right|=\operatorname{esssup}_{s \leq \tau_{u}}\left|\log \frac{\dot{\tilde{\lambda}}_{s}^{k} g\left(x_{s}\right)}{g_{k}\left(x_{\widetilde{\lambda}_{s}^{k}}^{k}\right)}\right| \underset{k \rightarrow \infty}{\longrightarrow} 0 .
\end{aligned}
$$

For the second part of the proposition we keep the same construction as previously. For any $v \geq 0$ we have that

$$
\tau_{\lambda_{u+v}^{k}}^{k}=\inf \left\{t \geq \lambda_{u}^{k} \mid \int_{\lambda_{u}^{k}}^{t} \frac{\mathrm{d} s}{g_{k}\left(x_{s}^{k}\right)} \geq v\right\} \wedge \tau_{\infty}^{k} .
$$

Using Fatou's lemma

$$
\liminf _{k \rightarrow \infty} \int_{\lambda_{u}^{k}}^{\left(\widetilde{\lambda}_{\tau_{\infty}}^{k}\right)^{+}} \frac{\mathrm{d} s}{g_{k}\left(x_{s}^{k}\right)}=\liminf _{k \rightarrow \infty} \int_{t}^{\tau_{\infty}+} \frac{\dot{\tilde{\lambda}}_{s}^{k} \mathrm{~d} s}{g_{k}\left(x_{\lambda_{s}^{k}}^{k}\right)} \geq \int_{t}^{\tau_{\infty}+} \frac{\mathrm{d} s}{g\left(x_{s}\right)}=\infty
$$

so, $\lim \sup _{k \rightarrow \infty} \tau_{\lambda_{u+v}^{k}}^{k}-\widetilde{\lambda}_{\tau_{\infty}}^{k} \leq 0$. Moreover, for $k$ large enough, $\tau_{\lambda_{u+v}^{k}}^{k} \geq \tau_{\lambda_{u}^{k}}^{k}=\widetilde{\lambda}_{t}^{k}$, so, $\lambda_{u+v}^{k}<\xi\left(g_{k} \cdot x^{k}\right)$ and

$$
\limsup _{k \rightarrow \infty} \sup _{u \leq w \leq u+v} d\left(g_{k} \cdot x_{\lambda_{w}^{k}}^{k},\left\{x_{s}\right\}_{t \leq s \leq \tau_{\infty}}\right)=0 .
$$


3.2. Connection between local and global Skorokhod topologies. Generally to take into account the explosion, one considers processes in $\mathbb{D}\left(S^{\Delta}\right)$, the set of cadlag processes described in Definition 2.1, associated to the space $S^{\Delta}$, and endowed with the global Skorokhod topology (see Definition 2.5). More precisely, the set of cadlag paths with values in $S^{\Delta}$ is given by

$$
\mathbb{D}\left(S^{\Delta}\right)=\left\{\begin{array}{l|l}
x \in\left(S^{\Delta}\right)^{\mathbb{R}_{+}} & \begin{array}{l}
\forall t \geq 0, x_{t}=\lim _{s \downarrow t} x_{s}, \text { and } \\
\forall t>0, x_{t-}:=\lim _{s \uparrow t} x_{s} \text { exists in } S^{\Delta}
\end{array}
\end{array}\right\} .
$$

A sequence $\left(x^{k}\right)_{k}$ in $\mathbb{D}\left(S^{\Delta}\right)$ converges to $x$ for the global Skorokhod topology if and only if there exists a sequence $\left(\lambda^{k}\right)_{k}$ of increasing homeomorphisms on $\mathbb{R}_{+}$such that

$$
\forall t \geq 0, \quad \lim _{k \rightarrow \infty} \sup _{s \leq t} d\left(x_{s}, x_{\lambda_{s}^{k}}^{k}\right)=0 \quad \text { and } \quad \lim _{k \rightarrow \infty}\left\|\lambda^{k}-\mathrm{id}\right\|_{t}=0 .
$$

In this section we give the connection between $\mathbb{D}\left(S^{\Delta}\right)$ with the global Skorokhod topology and $\mathbb{D}_{\text {loc }}(S)$ with the local Skorokhod topology.

We first identify these two measurable subspaces

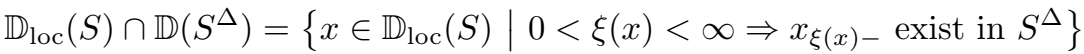

$$
\begin{aligned}
& =\left\{x \in \mathbb{D}\left(S^{\Delta}\right) \mid \forall t \geq \tau^{S}, x_{t}=\Delta\right\} .
\end{aligned}
$$

We can summarise our trajectories spaces by

$$
\begin{gathered}
\mathbb{D}(S) \subset \mathbb{D}_{\text {loc }}(S) \cap \mathbb{D}\left(S^{\Delta}\right) \quad \subset \mathbb{D}_{\text {loc }}(S) \subset \mathbb{D}_{\text {exp }}(S) . \\
\cap \\
\mathbb{D}\left(S^{\Delta}\right)
\end{gathered}
$$

Hence $\mathbb{D}_{\text {loc }}(S) \cap \mathbb{D}\left(S^{\Delta}\right)$ will be endowed with two topologies, the local topology from $\mathbb{D}_{\text {loc }}(S)$ and the global topology from $\mathbb{D}\left(S^{\Delta}\right)$.

Remark 3.8. 1) On $\mathbb{D}_{\text {loc }}(S) \cap \mathbb{D}\left(S^{\Delta}\right)$ the trace topology from $\mathbb{D}_{\text {loc }}(S)$ is weaker than the trace topology from $\mathbb{D}\left(S^{\Delta}\right)$. Eventually, these two topologies coincide on $\mathbb{D}(S)$. Indeed, this is clear using a metric $d$ on $S^{\Delta}$ and the characterisations of topologies given in Remark 2.6. The result in Corollary 3.10 below is a converse sentence of the present remark.

2) If $x \in \mathbb{D}_{\text {loc }}(S) \cap \mathbb{D}\left(S^{\Delta}\right)$ then $g \cdot x$ is well-defined in $\mathbb{D}_{\text {loc }}(S) \cap \mathbb{D}\left(S^{\Delta}\right)$ for

$$
g \in \mathrm{C}_{b}\left(S, \mathbb{R}_{+}^{*}\right) \subset\left\{g \in \mathrm{C}^{\neq 0}\left(S^{\Delta}, \mathbb{R}_{+}\right) \mid g(\Delta)=0\right\} .
$$

We deduce from Theorem 3.5 and the third point of Remark 3.6 that the mapping

$$
\begin{array}{ccc}
\mathrm{C}_{b}\left(S, \mathbb{R}_{+}^{*}\right) \times \mathbb{D}_{\text {loc }}(S) \cap \mathbb{D}\left(S^{\Delta}\right) & \rightarrow & \mathbb{D}_{\text {loc }}(S) \cap \mathbb{D}\left(S^{\Delta}\right) \\
(g, x) & \mapsto & g \cdot x
\end{array}
$$

is continuous between the topology of the uniform convergence and the global Skorokhod topology.

The following result is stated in a very general form because it will be useful when studying, for instance, the martingale problems.

Proposition 3.9 (Connection between $\mathbb{D}_{\text {loc }}(S)$ and $\mathbb{D}\left(S^{\Delta}\right)$ ). Let $E$ be an arbitrary locally compact Hausdorff space with countable base and consider

$$
\begin{array}{rlcc}
\mathbf{P}: \quad E & \rightarrow & \mathcal{P}\left(\mathbb{D}_{l o c}(S)\right) \\
a & \mapsto & \mathbf{P}_{a}
\end{array}
$$


a weakly continuous mapping for the local Skorokhod topology. Then for any open subset $U$ of $S$, there exists $g \in \mathrm{C}\left(S, \mathbb{R}_{+}\right)$such that $\{g \neq 0\}=U$, for all $a \in E$

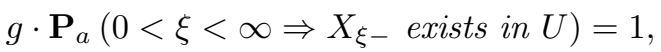

and the application

$$
\begin{aligned}
& g \cdot \mathbf{P}: \quad E \rightarrow \mathcal{P}\left(\left\{0<\xi<\infty \Rightarrow X_{\xi-} \text { exists in } U\right\}\right) \\
& a \mapsto \quad g \cdot \mathbf{P}_{a}
\end{aligned}
$$

is weakly continuous for the global Skorokhod topology from $\mathbb{D}\left(S^{\Delta}\right)$.

Before giving the proof of Proposition 3.9 we point out a direct application: we take $E:=\mathbb{N} \cup\{\infty\}, U=S$ and a sequence of Dirac probability measures $\mathbf{P}_{k}=\delta_{x^{k}}$, $\mathbf{P}_{\infty}=\delta_{x}$. Then we deduce from Proposition 3.9 the following:

Corollary 3.10 (Another description of $\mathbb{D}_{\text {loc }}(S)$ ). Let $x, x^{1}, x^{2}, \ldots \in \mathbb{D}_{\text {loc }}(S)$. Then the sequence $x^{k}$ converges to $x$ in $\mathbb{D}_{\text {loc }}(S)$, as $k \rightarrow \infty$, if and only if there exists $g \in \mathrm{C}\left(S, \mathbb{R}_{+}^{*}\right)$ such that $g \cdot x, g \cdot x^{1}, g \cdot x^{2}, \ldots \in \mathbb{D}\left(S^{\Delta}\right)$, and $g \cdot x^{k}$ converges to $g \cdot x$ in $\mathbb{D}\left(S^{\Delta}\right)$, as $k \rightarrow \infty$.

We proceed with the proof of Proposition 3.9 and, firstly we state a important result which will be our main tool:

Lemma 3.11. Let $D$ be a compact subset of $\mathbb{D}_{\text {loc }}(S)$ and $U$ be an open subset of S. There exists $g \in \mathrm{C}\left(S, \mathbb{R}_{+}\right)$such that:

i) $\{g \neq 0\}=U$,

ii) for all $x \in D,(g, x)$ is in the set $B_{\mathrm{tc}}$ given by (3.6) in Theorem 3.5 and

$$
g \cdot x \in\left\{0<\xi<\infty \Rightarrow X_{\xi-} \text { exists in } U\right\} .
$$

iii) the trace topologies of $\mathbb{D}_{\text {loc }}(S)$ and $\mathbb{D}\left(S^{\Delta}\right)$ coincide on $\{g \cdot x \mid x \in D\}$.

Furthermore, if $g \in \mathrm{C}\left(S, \mathbb{R}_{+}\right)$satisfies $\left.i\right)$-iii) and if $h \in \mathrm{C}\left(S, \mathbb{R}_{+}\right)$is such that $\{h \neq 0\}=U$ and $h \leq C g$ with a non-negative constant $C$, then $h$ also satisfies i)-iii).

Proof of Proposition 3.9: Let $\left(\widetilde{K}_{n}\right)_{n \in \mathbb{N}^{*}}$ be an increasing sequence of compact subset of $E$ such that $E=\bigcup_{n} \widetilde{K}_{n}$, then $\left\{\mathbf{P}_{a}\right\}_{a \in \widetilde{K}_{n}}$ is tight, for all $n \in \mathbb{N}^{*}$. So, there exist subsets $D_{n} \subset \mathbb{D}_{\text {loc }}(S)$ which are compacts for the topology of $\mathbb{D}_{\text {loc }}(S)$, and such that

$$
\sup _{a \in \widetilde{K}_{n}} \mathbf{P}_{a}\left(D_{n}^{c}\right) \leq \frac{1}{n}
$$

For any $n \in \mathbb{N}^{*}$, consider $g_{n}$ satisfying i)-iii) of Lemma 3.11 associated to the compact set $D_{n}$. It is no difficult to see that there exists $g \in \mathrm{C}\left(S, \mathbb{R}_{+}\right)$such that $\{g \neq 0\}=U$ and for all $n \in \mathbb{N}^{*}, g \leq C_{n} g_{n}$ for non-negative constants $C_{n}$. Hence, $g$ satisfies i)-iii) for all $D_{n}, n \in \mathbb{N}^{*}$. Hence, for all $a \in E$

$$
g \cdot \mathbf{P}_{a}\left(0<\xi<\infty \Rightarrow X_{\xi-} \text { exists in } U\right) \geq \mathbf{P}_{a}\left(\bigcup_{n \in \mathbb{N}^{*}} D_{n}\right)=1 .
$$

Let $a_{k}, a \in E$ such that $a_{k} \underset{k \rightarrow \infty}{\longrightarrow} a$. For $n$ large enough $\left\{a_{k}\right\}_{k} \subset \widetilde{K}_{n}$. Then, if $F$ is a subset of $\left\{0<\xi<\infty \Rightarrow X_{\xi}\right.$ - exists in $\left.U\right\}$ which is closed for the topology of 


\section{$\mathbb{D}\left(S^{\Delta}\right)$}

$$
\begin{aligned}
\limsup _{k \rightarrow \infty} g \cdot \mathbf{P}_{a_{k}}(F)-g \cdot \mathbf{P}_{a}(F) & \\
& \leq \limsup _{k \rightarrow \infty} \mathbf{P}_{a_{k}}\left(X \in D_{n}, g \cdot X \in F\right)-\mathbf{P}_{a}\left(X \in D_{n}, g \cdot X \in F\right)+\frac{1}{n} .
\end{aligned}
$$

But thanks to iii) in Lemma 3.11, $\left\{X \in D_{n}, g \cdot X \in F\right\}$ is a subset of $\mathbb{D}_{\text {loc }}(S)$ which is closed for the topology of $\mathbb{D}_{\text {loc }}(S)$. Hence, by using the Portmanteau theorem (see for instance Theorem 2.1 from Billingsley, 1999, p. 16)

$$
\limsup _{k \rightarrow \infty} \mathbf{P}_{a_{k}}\left(X \in D_{n}, g \cdot X \in F\right) \leq \mathbf{P}_{a}\left(X \in D_{n}, g \cdot X \in F\right)
$$

and so, letting $n \rightarrow \infty$,

$$
\limsup _{k \rightarrow \infty} g \cdot \mathbf{P}_{a_{k}}(F) \leq g \cdot \mathbf{P}_{a}(F) .
$$

By using the Portmanteau theorem, the proof of the proposition is complete, except for the proof of Lemma 3.11.

Proof of Lemma 3.11: Let $d$ be a metric on $S^{\Delta}$ and denote

$$
K_{n}:=\left\{a \in S \mid d\left(a, S^{\Delta} \backslash U\right) \geq 2^{-n}\right\} .
$$

By using Theorem 2.8,there exists a sequence $\left(\eta_{n}\right)_{n} \in(0,1)^{\mathbb{N}}$ decreasing to 0 such that

$$
\sup _{x \in D} \omega_{2^{n}, B\left(\Delta, 2^{-n-2}\right)^{c}, x}^{\prime}\left(\eta_{n}\right)<2^{-n-2} .
$$

Moreover, there exists $g \in \mathrm{C}\left(S^{\Delta},[0,1]\right)$ such that $\{g \neq 0\}=U$ and $g_{\mid K_{n}^{c}} \leq 2^{-n} \eta_{n}$. For instance, we can define

$$
g(a):=\sup _{n \geq 0}\left(\left(2^{-n} \eta_{n}\right) \wedge d\left(a, S^{\Delta} \backslash K_{n}\right)\right), a \in S^{\Delta} .
$$

Let $x \in D$ be. We consider the following two situations:

- If $\tau_{\infty}^{g}(x)<\infty$ and $\left\{x_{s}\right\}_{s<\tau_{\infty}^{g}(x)}$ is not a compact of $U$, take $m \in \mathbb{N}$ such that $2^{m} \geq \tau_{\infty}^{g}(x)$, denote

$$
t:=\min \left\{s \geq 0 \mid x_{s} \notin \stackrel{\circ}{K}_{m+1}\right\}<\tau_{\infty}^{g}(x)
$$

and let $n \geq m$ be such that $x_{t} \in K_{n+2} \backslash \stackrel{\circ}{K}_{n+1}$. Using (3.9) there exist $t_{1}, t_{2} \in \mathbb{R}_{+}$such that $t_{1} \leq t<t_{2}<\tau_{\infty}^{g}(x), t_{2}-t_{1}>\eta_{n}$ and $x_{s} \notin K_{n}$ for all $s \in\left[t_{1}, t_{2}\right)$. So,

$$
A_{\tau_{\infty}^{g}(x)}^{g}(x) \geq \int_{t_{1}}^{t_{2}} \frac{\mathrm{d} s}{g\left(x_{s}\right)} \geq 2^{m}
$$

hence, letting $m$ goes to infinity,

$$
A_{\tau_{\infty}^{g}(x)}^{g}(x)=\infty .
$$

- If $\tau_{\infty}^{g}(x)<\xi(x)$ and $g\left(x_{\tau_{\infty}^{g}(x)-}\right) \neq 0$, then $g\left(x_{\tau_{\infty}^{g}(x)}\right)=0$. Let $m \in \mathbb{N}$ be such that $2^{m} \geq \tau_{\infty}^{g}(x)$ and $\left\{x_{s}\right\}_{s \leq \tau_{\infty}^{g}(x)} \subset B\left(\Delta, 2^{-m-2}\right)^{c}$. Using (3.9), 
there exist $t_{1}, t_{2} \in \mathbb{R}_{+}$such that $t_{1} \leq \tau_{\infty}^{g}(x)<t_{2}<\xi(x), t_{2}-t_{1}>\eta_{m}$ and $x_{s} \notin K_{m}$ for all $s \in\left[t_{1}, t_{2}\right)$. So

$$
\int_{0}^{\tau_{\infty}^{g}(x)+\eta_{m}} \frac{\mathrm{d} s}{g\left(x_{s}\right)} \geq \int_{t_{1}}^{t_{1}+\eta_{m}} \frac{\mathrm{d} s}{g\left(x_{s}\right)} \geq 2^{m}
$$

hence letting $m$ tend to infinity

$$
\int_{0}^{\tau_{\infty}^{g}(x)+} \frac{\mathrm{d} s}{g\left(x_{s}\right)}=\infty
$$

Hence, we obtain that $(g, x) \in B_{\text {tc }}$ and $g \cdot x \in\left\{0<\xi<\infty \Rightarrow X_{\xi-}\right.$ exists in $\left.U\right\}$ and ii) is verified.

We proceed by proving iii). Thanks to Remark 3.8, to get the equivalence of the topologies it is enough to prove that if $x^{k}, x \in D$ are such that $g \cdot x^{k} \rightarrow g \cdot x$ for the topology from $\mathbb{D}_{\text {loc }}(S)$ and $\xi(g \cdot x)<\infty$, then the convergence also holds for the topology from $\mathbb{D}\left(S^{\Delta}\right)$. Let $\lambda^{k} \in \widetilde{\Lambda}$ be such that

$$
\sup _{s \leq \xi(g \cdot x)} d\left(g \cdot x_{s}, g \cdot x_{\lambda_{s}^{k}}^{k}\right) \longrightarrow 0, \quad\left\|\log \dot{\lambda}^{k}\right\|_{\xi(g \cdot x)} \longrightarrow 0, \quad \text { as } k \rightarrow \infty .
$$

We may suppose that $\dot{\lambda}_{s}^{k}=0$, for $s \geq \xi(g \cdot x)$. Denote $t_{k}:=\lambda_{\xi(g \cdot x)}^{k}$ and choose $m \in \mathbb{N}$ be such that $\left\{g \cdot x_{s}\right\}_{s<\xi(g \cdot x)} \Subset \stackrel{\circ}{K}_{m}$ and $\xi(g \cdot x)<2^{m}$. Then, for $k$ large enough $\left\{g \cdot x_{s}^{k}\right\}_{s<t_{k}} \Subset \stackrel{\circ}{K_{m}}, g \cdot x_{t_{k}}^{k} \notin K_{m+1}$ and $t_{k}<2^{m}$.

- Either $g \cdot x_{t_{k}}^{k} \notin U$ and so $g \cdot x_{\lambda_{s}^{k}}^{k}=g \cdot x_{t^{k}}^{k}$ for all $s \geq \xi(g \cdot x)$.

- Or $g \cdot x_{t_{k}}^{k} \in U$ and let $n \geq m$ be such that $g \cdot x_{t_{k}}^{k}=x_{\tau_{t_{k}}^{g}\left(x^{k}\right)} \in K_{n+2} \backslash \stackrel{\circ}{K}_{n+1}$. Using (3.9), $d\left(x_{s}, x_{t_{k}}^{k}\right)<2^{-n-2}$, and so, $x_{s} \in U \backslash K_{n}$, for all $s \in$ $\left[\tau_{t_{k}}^{g}\left(x^{k}\right), \tau_{t_{k}}^{g}\left(x^{k}\right)+\eta_{n}\right]$. Hence, $A_{\tau_{t_{k}}^{g}\left(x^{k}\right)+\eta_{n}}^{g} \geq t_{k}+2^{n}$, so $d\left(g \cdot x_{s}, g \cdot x_{t_{k}}^{k}\right)<$ $2^{-n-2}$ for all $s \in\left[t_{k}, t_{k}+2^{n}\right]$.

Hence we obtain that, for $k$ large enough,

$$
\sup _{s \leq \xi(g \cdot x)+2^{m}} d\left(g \cdot x_{s}, g \cdot x_{\lambda_{s}^{k}}^{k}\right) \leq \sup _{s \leq \xi(g \cdot x)} d\left(g \cdot x_{s}, g \cdot x_{\lambda_{s}^{k}}^{k}\right)+2^{-m-2},
$$

so letting $m$ goes to the infinity we obtain that $g \cdot x^{k}$ converge to $g \cdot x$ for the global Skorokhod topology from $\mathbb{D}\left(S^{\Delta}\right)$. Hence, the proof of iii) is done.

Finally, to prove the last part of the lemma let $g \in \mathrm{C}\left(S, \mathbb{R}_{+}\right)$be such that i)-iii) are satisfied and let $h \in \mathrm{C}\left(S, \mathbb{R}_{+}\right)$be such that $\{h \neq 0\}=U$ and $h \leq C g$ with a non-negative constant $C$. Thanks to Remark 3.6, $(h, x)$ belongs to the set $B_{\text {tc }}$ given by (3.6), it is also clear that $h \cdot x \in\left\{0<\xi<\infty \Rightarrow X_{\xi-}\right.$ exists in $\left.U\right\}$. We have that $\frac{h}{g} \in \mathrm{C}_{b}\left(U, \mathbb{R}_{+}^{*}\right)$, so using (3.7) for $S$ and $S^{\Delta}$, the bijection

$$
\begin{array}{ccc}
\{g \cdot x \mid x \in D\} & \rightarrow\{h \cdot x \mid x \in D\} \\
x & \mapsto & \frac{h}{g} \cdot x
\end{array}
$$

is continuous for the topology of $\mathbb{D}_{\text {loc }}(S)$, but also of $\mathbb{D}\left(S^{\Delta}\right)$. But since $\{g \cdot x \mid x \in D\}$ is compact, this application is bi-continuous, and we obtain the result. Now the proof of lemma is complete. 


\section{Proofs of main results on local Skorokhod metrics}

In this section we will prove Theorem 2.4 and Theorem 2.8, by following the strategy developed in $§ 12$, pp. 121-137 from Billingsley (1999). To construct metrics on $\mathbb{D}_{\text {loc }}(S)$, we will consider a metric $d$ on $S$. To begin with, we define two families of pseudo-metrics:

Lemma 4.1 (Skorokhod metrics). For $0 \leq t<\infty$ and $K \subset S$ a compact subset, the following two expressions on $\mathbb{D}_{\exp }(S)$ :

$$
\begin{aligned}
\rho_{t, K}\left(x^{1}, x^{2}\right):= & \inf _{\substack{t_{i} \leq \xi\left(x^{i}\right) \\
\lambda \in \Lambda, \lambda_{t_{1}}=t_{2}}} \sup _{s<t_{1}} d\left(x_{s}^{1}, x_{\lambda_{s}}^{2}\right) \vee\|\lambda-\mathrm{id}\|_{t_{1}} \\
\widetilde{\rho}_{t, K}\left(x^{1}, x^{2}\right):= & \inf _{\substack{t_{i} \leq \xi\left(x^{i}\right) \\
\lambda \in \widetilde{\Lambda}, \lambda_{t_{1}}=t_{2}}} \sup _{s<t_{1}} d\left(x_{s}^{1}, x_{\lambda_{s}}^{2}\right) \vee\|\log \dot{\lambda}\|_{t_{1}} \vee\|\lambda-\mathrm{id}\|_{t_{1}} \\
& \vee \max _{i \in\{1,2\}}\left(d\left(x_{t_{i}}^{i}, K^{c}\right) \wedge\left(t-t_{i}\right)_{+} \mathbb{1}_{t_{i}<\xi\left(x^{i}\right)}\right), \\
&
\end{aligned}
$$

define two pseudo-metrics.

Proof: Let us perform the proof for $\widetilde{\rho}_{t, K}$, the proof being similar for $\rho_{t, K}$. The non-trivial part is the triangle inequality. Let $x^{1}, x^{2}, x^{3} \in \mathbb{D}_{\exp }(S)$ and $\varepsilon>0$ be then there are $t_{1} \leq \xi\left(x^{1}\right), t_{2}, \widehat{t_{2}} \leq \xi\left(x^{2}\right), \widehat{t_{3}} \leq \xi\left(x^{3}\right)$ and $\lambda^{1} \in \widetilde{\Lambda}, \lambda^{2} \in \widetilde{\Lambda}$ such that

$$
\begin{aligned}
\tilde{\rho}_{t, K}\left(x^{1}, x^{2}\right)+\varepsilon \geq \sup _{s<t_{1}} d\left(x_{s}^{1}, x_{\lambda_{s}^{1}}^{2}\right) \vee & \left\|\log \dot{\lambda}^{1}\right\|_{t_{1}} \vee\left\|\lambda^{1}-\mathrm{id}\right\|_{t_{1}} \\
& \vee \max _{i \in\{1,2\}}\left(d\left(x_{t_{i}}^{i}, K^{c}\right) \wedge\left(t-t_{i}\right)_{+} \mathbb{1}_{t_{i}<\xi\left(x^{i}\right)}\right), \\
\tilde{\rho}_{t, K}\left(x^{2}, x^{3}\right)+\varepsilon \geq \sup _{s<\widehat{t}_{2}} d\left(x_{s}^{2}, x_{\lambda_{s}^{2}}^{3}\right) \vee & \left\|\log \dot{\lambda}^{2}\right\|_{\widehat{t}_{2}} \vee\left\|\lambda^{2}-\mathrm{id}\right\|_{\widehat{t}_{2}} \\
& \vee \max _{i \in\{2,3\}}\left(d\left(x_{\widehat{t}_{i}}^{i}, K^{c}\right) \wedge\left(t-\widehat{t}_{i}\right)_{+} \mathbb{1}_{\widehat{t}_{i}<\xi\left(x^{i}\right)}\right) .
\end{aligned}
$$

Define $\check{t}_{2}:=t_{2} \wedge \widehat{t}_{2}, \check{t}_{1}:=\left(\lambda^{1}\right)_{\breve{t}_{2}}^{-1}, \check{t}_{3}:=\lambda_{\check{t}_{2}}^{2}$ and $\lambda:=\lambda^{2} \circ \lambda^{1}$. Then

$$
\begin{aligned}
& \sup _{s<\check{t}_{1}} d\left(x_{s}^{1}, x_{\lambda_{s}}^{3}\right) \leq \sup _{s<t_{1}} d\left(x_{s}^{1}, x_{\lambda_{s}^{1}}^{2}\right)+\sup _{s<\widehat{t}_{2}} d\left(x_{s}^{2}, x_{\lambda_{s}^{2}}^{3}\right), \\
& \|\log \dot{\lambda}\|_{\check{t}_{1}} \leq\left\|\log \dot{\lambda}^{1}\right\|_{t_{1}}+\left\|\log \dot{\lambda}^{2}\right\|_{\widehat{t}_{2}}, \quad\|\lambda-\mathrm{id}\|_{\check{t}_{1}} \leq\left\|\lambda^{1}-\mathrm{id}\right\|_{t_{1}}+\left\|\lambda^{2}-\mathrm{id}\right\|_{\widehat{t}_{2}} .
\end{aligned}
$$

Moreover, for instance, if $\check{t}_{1} \neq t_{1}$, then $\check{t}_{1}<t_{1} \leq \xi\left(x_{1}\right), \widehat{t}_{2}=\check{t}_{2}<t_{2} \leq \xi\left(x_{2}\right)$ and

$$
\begin{aligned}
d\left(x_{\check{t}_{1}}^{1}, K^{c}\right) \wedge\left(t-\check{t}_{1}\right)_{+} & \leq d\left(x_{\check{t}_{1}}^{1}, x_{\check{t}_{2}}^{2}\right) \vee\left|\check{t}_{2}-\check{t}_{1}\right|+d\left(x_{\check{t}_{2}}^{2}, K^{c}\right) \wedge\left(t-\check{t}_{2}\right)_{+} \\
& \leq \sup _{s<t_{1}} d\left(x_{s}^{1}, x_{\lambda_{s}^{1}}^{2}\right) \vee\left\|\lambda^{1}-\mathrm{id}\right\|_{t_{1}}+d\left(x_{\widehat{t}_{2}}^{2}, K^{c}\right) \wedge\left(t-\widehat{t}_{2}\right)_{+} .
\end{aligned}
$$

Hence

$$
\widetilde{\rho}_{t, K}\left(x^{1}, x^{3}\right) \leq \widetilde{\rho}_{t, K}\left(x^{1}, x^{2}\right)+\widetilde{\rho}_{t, K}\left(x^{2}, x^{3}\right)+2 \varepsilon,
$$

so letting $\varepsilon \rightarrow 0$, we obtain the triangular inequality.

We prove that these pseudo-metrics are in somehow equivalent:

Lemma 4.2. Take $x, y \in \mathbb{D}_{\text {loc }}(S), t \geq 0$ and a compact subset $K \subset S$, if $\rho_{t, K}(x, y) \leq \frac{1}{9}$ then

$$
\widetilde{\rho}_{t, K}(x, y) \leq 6 \cdot \sqrt{\rho_{t, K}(x, y)} \vee \omega_{t, K, x}^{\prime}\left(\sqrt{\rho_{t, K}(x, y)}\right) .
$$


Proof: Let $\varepsilon>0$ be arbitrary. There exist $\mu \in \Lambda$ and $T \geq 0$ such that $T \leq \xi(x)$, $\mu_{T} \leq \xi(y)$ and

$$
\begin{aligned}
\sup _{s<T} d\left(x_{s}, y_{\mu_{s}}\right) \vee\|\mu-\mathrm{id}\|_{T} & \leq \rho_{t, K}(x, y)+\varepsilon, \\
d\left(x_{T}, K^{c}\right) \wedge(t-T)_{+} \mathbb{1}_{T<\xi(x)} & \leq \rho_{t, K}(x, y)+\varepsilon, \\
d\left(y_{\mu_{T}}, K^{c}\right) \wedge\left(t-\mu_{T}\right)_{+} \mathbb{1}_{\mu_{T}<\xi(y)} & \leq \rho_{t, K}(x, y)+\varepsilon .
\end{aligned}
$$

Let $\delta>2 \rho_{t, K}(x, y)+2 \varepsilon$ be arbitrary, there exist $0=t_{0}<\cdots<t_{N} \leq \xi(x)$ such that

$$
\sup _{\substack{0 \leq i<N \\ t_{i} \leq s_{1}, s_{2}<t_{i+1}}} d\left(x_{s_{1}}, x_{s_{2}}\right) \leq \omega_{t, K, x}^{\prime}(\delta)+\varepsilon
$$

$\delta<t_{i+1}-t_{i} \leq 2 \delta$ and $\left(t_{N}, x_{t_{N}}\right) \notin[0, t] \times K$. Set $n_{0}:=\max \left\{0 \leq i \leq N \mid t_{i} \leq T\right\}$ and $\widetilde{T}:=t_{n_{0}}$. Define $\lambda \in \widetilde{\Lambda}$ by

$$
\begin{cases}\forall i \leq n_{0}, & \lambda_{t_{i}}=\mu_{t_{i}}, \\ \forall i<n_{0}, & \lambda \text { is affine on }\left[t_{i}, t_{i+1}\right], \\ \forall s \geq \widetilde{T}, & \dot{\lambda}_{s}=1 .\end{cases}
$$

Then

$$
\|\lambda-\mathrm{id}\|=\sup _{0<i \leq n_{0}}\left\|\mu_{t_{i}}-t_{i}\right\| \leq\|\mu-\mathrm{id}\|_{T} \leq \rho_{t, K}(x, y)+\varepsilon .
$$

For $0 \leq i<n_{0}$ we have

$$
\left|\frac{\mu_{t_{i+1}}-\mu_{t_{i}}-t_{i+1}+t_{i}}{t_{i+1}-t_{i}}\right| \leq \frac{2\|\mu-\mathrm{id}\|_{T}}{\delta} \leq \frac{2 \rho_{t, K}(x, y)+2 \varepsilon}{\delta}<1,
$$

so, by the classical estimate:

$$
|\log (1+r)| \leq \frac{|r|}{1-|r|} \quad \text { for }|r|<1 .
$$

we deduce

$$
\|\log \dot{\lambda}\|=\sup _{0 \leq i<n_{0}}\left|\log \frac{\mu_{t_{i+1}}-\mu_{t_{i}}}{t_{i+1}-t_{i}}\right| \leq \frac{2 \rho_{t, K}(x, y)+2 \varepsilon}{\delta-2 \rho_{t, K}(x, y)-2 \varepsilon} .
$$

Since for $s<\lambda_{\widetilde{T}}, \lambda_{s}^{-1}$ and $\mu_{s}^{-1}$ lies in the same interval $\left[t_{i}, t_{i+1}\right)$. Therefore,

$$
\sup _{s<\lambda_{\widetilde{T}}} d\left(x_{\lambda_{s}^{-1}}, y_{s}\right) \leq \sup _{s<\lambda_{\widetilde{T}}}\left(d\left(x_{\mu_{s}^{-1}}, y_{s}\right)+d\left(x_{\mu_{s}^{-1}}, x_{\lambda_{s}^{-1}}\right)\right) \leq \rho_{t, K}(x, y)+\omega_{t, K, x}^{\prime}(\delta)+2 \varepsilon .
$$

For the two last terms in $\widetilde{\rho}_{t, K}$ we may consider only the case were $\widetilde{T} \neq T$. If $n_{0}=N: d\left(x_{\widetilde{T}}, K^{c}\right) \wedge(t-\widetilde{T})_{+}=0$, otherwise:

$$
\begin{aligned}
d\left(x_{\widetilde{T}}, K^{c}\right) \wedge(t-\widetilde{T})_{+} & \leq d\left(x_{T}, K^{c}\right) \wedge(t-T)_{+}+d\left(x_{\widetilde{T}}, x_{T}\right) \vee(T-\widetilde{T}) \\
& \leq \rho_{t, K}(x, y)+\omega_{t, K, x}^{\prime}(\delta) \vee(2 \delta)+2 \varepsilon .
\end{aligned}
$$

By using $\lambda_{\widetilde{T}}=\mu_{\widetilde{T}}$, we also have

$$
\begin{aligned}
d\left(y_{\lambda_{\widetilde{T}}}, K^{c}\right) \wedge\left(t-\lambda_{\widetilde{T}}\right)_{+} & \leq d\left(x_{\widetilde{T}}, K^{c}\right) \wedge(t-\widetilde{T})_{+}+d\left(x_{\widetilde{T}}, y_{\mu_{\widetilde{T}}}\right) \vee\left|\widetilde{T}-\mu_{\widetilde{T}}\right| \\
& \leq 2 \rho_{t, K}(x, y)+\omega_{t, K, x}^{\prime}(\delta) \vee(2 \delta)+3 \varepsilon .
\end{aligned}
$$

Letting $\varepsilon \rightarrow 0$ we obtain that for all $\delta>2 \rho_{t, K}(x, y)$,

$$
\tilde{\rho}_{t, K}(x, y) \leq\left(2 \rho_{t, K}(x, y)+\omega_{t, K, x}^{\prime}(\delta) \vee(2 \delta)\right) \vee \frac{2 \rho_{t, K}(x, y)}{\delta-2 \rho_{t, K}(x, y)}
$$


Finally, by taking $\delta:=\sqrt{\rho_{t, K}(x, y)}$ we have for $\rho_{t, K}(x, y) \leq \frac{1}{9}$

$$
\begin{aligned}
& \widetilde{\rho}_{t, K}(x, y) \leq\left(2 \rho_{t, K}(x, y)+\omega_{t, K, x}^{\prime}(\delta) \vee(2 \delta)\right) \vee \frac{2 \rho_{t, K}(x, y)}{\delta-2 \rho_{t, K}(x, y)} \\
& \leq\left(\frac{2}{3} \sqrt{\rho_{t, K}(x, y)}+\omega_{t, K, x}^{\prime}\left(\sqrt{\rho_{t, K}(x, y)}\right) \vee\left(2 \sqrt{\rho_{t, K}(x, y)}\right)\right) \vee 6 \sqrt{\rho_{t, K}(x, y)} \\
& \leq 6 \cdot \sqrt{\rho_{t, K}(x, y)} \vee \omega_{t, K, x}^{\prime}\left(\sqrt{\rho_{t, K}(x, y)}\right) .
\end{aligned}
$$

At this level it can be pointed out that we obtain the definition of the local Skorokhod topology. Indeed, by using Proposition 2.7, Lemma 4.2 and the fact that $\rho_{t, K} \leq \widetilde{\rho}_{t, K}$, the two families of pseudo-metrics $\left(\rho_{t, K}\right)_{t, K}$ and $\left(\widetilde{\rho}_{t, K}\right)_{t, K}$ define the same topology on $\mathbb{D}_{\text {loc }}(S)$, the local Skorokhod topology.

If $\left(K_{n}\right)_{n \in \mathbb{N}}$ is an exhaustive sequence of compact sets of $S$, then the mapping

$$
\begin{array}{ccc}
\mathbb{D}_{\text {loc }}(S)^{2} & \rightarrow & \mathbb{R}_{+} \\
(x, y) & \mapsto & \sum_{n \in \mathbb{N}} 2^{-n} \widetilde{\rho}_{n, K_{n}}(x, y) \wedge 1
\end{array}
$$

is a metric for the local Skorokhod topology. By using a diagonal extraction procedure, it is not difficult to prove that a sequence $\left(x^{k}\right)_{k}$ converges to $x$ for this topology if and only if there exists a sequence $\left(\lambda^{k}\right)_{k}$ in $\widetilde{\Lambda}$ such that

- either $\xi(x)<\infty$ and $\left\{x_{s}\right\}_{s<\xi(x)} \Subset S$ : for $k$ large enough $\lambda_{\xi(x)}^{k} \leq \xi\left(x^{k}\right)$ and $\sup _{s<\xi(x)} d\left(x_{s}, x_{\lambda_{s}^{k}}^{k}\right) \longrightarrow 0, \quad \quad x_{\lambda_{\xi(x)}^{k}}^{k} \longrightarrow \Delta, \quad\left\|\log \dot{\lambda}^{k}\right\|_{\xi(x)} \longrightarrow 0, \quad$ as $k \rightarrow \infty$,

- or $\xi(x)=\infty$ or $\left\{x_{s}\right\}_{s<\xi(x)} \notin S$ : for all $t<\xi(x)$, for $k$ large enough $\lambda_{t}^{k}<\xi\left(x^{k}\right)$ and

$$
\sup _{s \leq t} d\left(x_{s}, x_{\lambda_{s}^{k}}^{k}\right) \longrightarrow 0, \quad\left\|\log \dot{\lambda}^{k}\right\|_{t} \longrightarrow 0, \quad \text { as } k \rightarrow \infty .
$$

The local Skorokhod topology can be described by a similar characterisation with $\lambda^{k} \in \widetilde{\Lambda}$ replaced by $\lambda^{k} \in \Lambda$ and respectively, $\left\|\log \dot{\lambda}^{k}\right\|$ replaced by $\| \lambda-$ id $\|$. The fact that the local Skorokhod topology does not depend on the distance $d$ is a consequence of the following lemma, which states essentially that two metrics on a compact set are uniformly equivalent:

Lemma 4.3. Let $T$ be a set and $x, x^{k} \in S^{T}$ be such that $\left\{x_{t}\right\}_{t \in T} \Subset S$, then

$$
\sup _{t \in T} d\left(x_{t}, x_{t}^{k}\right) \longrightarrow 0, \quad \text { as } k \rightarrow \infty
$$

if and only if

$\forall U \subset S^{2}$ open subset containing $\{(y, y)\}_{y \in S}, \quad \exists k_{0} \forall k \geq k_{0}, \forall t \in T,\left(x_{t}, x_{t}^{k}\right) \in U$.

So the topology of the uniform convergence on $\left\{x \in S^{T} \mid\left\{x_{t}\right\}_{t \in T} \Subset S\right\}$ depends only of the topology of $S$.

Proof: Suppose that $\sup _{t \in T} d\left(x_{t}, x_{t}^{k}\right) \longrightarrow 0$ as $k \rightarrow \infty$ and take an open subset $U \subset S^{2}$ containing $\{(y, y)\}_{y \in S}$. By compactness there exists $\varepsilon>0$ such that

$$
\left\{\left(y_{1}, y_{2}\right) \in S^{2} \mid y_{1} \in\left\{x_{t}\right\}_{t}, d\left(y_{1}, y_{2}\right)<\varepsilon\right\} \subset U
$$

so, for $k$ large enough and for all $t,\left(x_{t}, x_{t}^{k}\right) \in U$. To get the converse property it suffices to consider, for each $\varepsilon>0$, the open set $U_{\varepsilon}:=\left\{\left(y_{1}, y_{2}\right) \in S^{2} \mid d\left(y_{1}, y_{2}\right)<\varepsilon\right\}$ which clearly contains $\{(y, y)\}_{y \in S}$. 
In the next lemma we discuss the completeness :

Lemma 4.4. Suppose that $(S, d)$ is complete. Then any sequence $\left(x^{k}\right)_{k} \in$ $\left(\mathbb{D}_{\text {loc }}(S)\right)^{\mathbb{N}}$ satisfying

$$
\forall t \geq 0, \forall K \subset S \text { compact, } \quad \widetilde{\rho}_{t, K}\left(x^{k_{1}}, x^{k_{2}}\right) \underset{k_{1}, k_{2} \rightarrow \infty}{\longrightarrow} 0,
$$

admits a limit for the local Skorokhod topology.

The proof of this lemma follows from the same reasoning as the proof of the triangular inequality, the proof of Theorem 12.2 pp. 128-129 from Billingsley (1999) and the proof of Theorem 5.6 pp. 121-122 from Ethier and Kurtz (1986).

Proof: It suffices to prove that $\left(x^{k}\right)_{k}$ have a converging subsequence. By taking, possibly, a subsequence we can suppose that

$$
\forall t \geq 0, \forall K \subset S \text { compact, } \quad \sum_{k \geq 0} \widetilde{\rho}_{t, K}\left(x^{k}, x^{k+1}\right)<\infty .
$$

We split our proof in five steps.

Step 1: we construct a sequence $\left(\lambda^{k}\right)_{k} \subset \widetilde{\Lambda}$. Let $\mu^{k} \in \widetilde{\Lambda}$ and $\widetilde{t}_{k} \geq 0$ be such that for all $t \geq 0$ and $K \subset S$ compact, we have for $k$ large enough

$$
\begin{array}{r}
\widetilde{t}_{k} \leq \xi\left(x^{k}\right), \quad \mu_{\widetilde{t}_{k}}^{k} \leq \xi\left(x^{k+1}\right), \\
\sup _{s<\widetilde{t}_{k}} d\left(x_{s}^{k}, x_{\mu_{s}^{k}}^{k+1}\right) \vee\left\|\log \dot{\mu}^{k}\right\|_{\widetilde{t}_{k}} \vee\left\|\mu^{k}-\mathrm{id}\right\|_{\widetilde{t}_{k}} \leq 2 \widetilde{\rho}_{t, K}\left(x^{k}, x^{k+1}\right), \\
\left(d\left(x_{\widetilde{t}_{k}}^{k}, K^{c}\right) \wedge\left(t-\widetilde{t}_{k}\right)_{+} \mathbb{1}_{\widetilde{t}_{k}<\xi\left(x^{k}\right)}\right) \leq 2 \widetilde{\rho}_{t, K}\left(x^{k}, x^{k+1}\right), \\
\left(d\left(x_{\tilde{t}_{k}}^{k+1}, K^{c}\right) \wedge\left(t-\mu_{\widetilde{t}_{k}}^{k}\right)_{+} \mathbb{1}_{\mu_{\widetilde{t}_{k}}^{k}<\xi\left(x^{k+1}\right)}\right) \leq 2 \widetilde{\rho}_{t, K}\left(x^{k}, x^{k+1}\right) .
\end{array}
$$

For all $k \geq 0$ define

$$
t_{k}:=\bigwedge_{i \geq 0}\left(\mu^{k}\right)^{-1} \circ \cdots \circ\left(\mu^{k+i-1}\right)^{-1}\left(\widetilde{t}_{k+i}\right),
$$

so, $t_{k} \leq \widetilde{t}_{k}$ and $\mu_{t_{k}}^{k} \leq t_{k+1}$. For $k, i \geq 0$

$$
\begin{aligned}
\left\|\log \frac{\mathrm{d}}{\mathrm{d} s}\left(\mu^{k+i-1} \circ \cdots \circ \mu^{k}(s)\right)\right\|_{t_{k}} & \leq \sum_{\ell=k}^{k+i-1}\left\|\log \dot{\mu}^{\ell}\right\|_{\widetilde{t}_{\ell}}, \\
\left\|\mu^{k+i-1} \circ \cdots \circ \mu^{k}-\mathrm{id}\right\|_{t_{k}} & \leq \sum_{\ell=k}^{k+i-1}\left\|\mu^{\ell}-\mathrm{id}\right\|_{\widetilde{t}_{\ell}}
\end{aligned}
$$

and for $j \geq i$

$$
\begin{aligned}
\left\|\mu^{k+j-1} \circ \cdots \circ \mu^{k}-\mu^{k+i-1} \circ \cdots \circ \mu^{k}\right\|_{t_{k}} & \leq\left\|\mu^{k+j-1} \circ \cdots \circ \mu^{k+i}-\mathrm{id}\right\|_{t_{k+i}} \\
& \leq \sum_{\ell=k+i}^{k+j-1}\left\|\mu^{\ell}-\mathrm{id}\right\|_{\widetilde{t}_{\ell}} .
\end{aligned}
$$

Using (4.2) and (4.3) we obtain

$$
\sum_{\ell \geq 0}\left\|\mu^{\ell}-\mathrm{id}\right\|_{\widetilde{t}_{\ell}}<\infty, \quad \sum_{\ell \geq 0}\left\|\log \dot{\mu}^{\ell}\right\|_{\widetilde{t}_{\ell}}<\infty
$$


so, the restriction to $\left[0, t_{k}\right]$ of continuous functions $\mu^{k+i-1} \circ \cdots \circ \mu^{k}$ converges uniformly to a continuous function. Set

$$
\begin{cases}\lambda_{s}^{k}:=\lim _{i \rightarrow \infty} \mu^{k+i-1} \circ \cdots \circ \mu^{k}(s), & \text { if } s \leq t_{k} \\ \dot{\lambda}_{s}^{k}:=1, & \text { if } s \geq t_{k} .\end{cases}
$$

Clearly for $s \leq t_{k}, \lambda_{s}^{k}=\lambda^{k+1} \circ \mu^{k}(s)$. We have

$$
\begin{aligned}
\left\|\lambda^{k}-\mathrm{id}\right\| & \leq \sup _{i \geq 0}\left\|\mu^{k+i-1} \circ \cdots \circ \mu^{k}-\mathrm{id}\right\|_{t_{k}} \leq \sum_{\ell \geq k}\left\|\mu^{\ell}-\mathrm{id}\right\|_{\tilde{t}_{\ell}}<\infty \\
\left\|\log \dot{\lambda}^{k}\right\| & =\sup _{0 \leq s_{1}<s_{2} \leq t_{k}}\left|\log \frac{\lambda_{s_{2}}^{k}-\lambda_{s_{1}}^{k}}{s_{2}-s_{1}}\right| \leq \sup _{i \geq 0}\left\|\log \frac{\mathrm{d}}{\mathrm{d} s}\left(\mu^{k+i-1} \circ \cdots \circ \mu^{k}(s)\right)\right\|_{t_{k}} \\
& \leq \sum_{\ell \geq k}\left\|\log \dot{\mu}^{\ell}\right\|_{\tilde{t}_{\ell}}<\infty
\end{aligned}
$$

so, $\lambda^{k} \in \widetilde{\Lambda}$.

Step 2: we construct a path $x \in \mathbb{D}_{\text {exp }}(S)$. For all $k \geq 0$ : $\lambda_{t_{k}}^{k}=\lambda_{\mu_{t_{k}}^{k}}^{k+1} \leq \lambda_{t_{k+1}}^{k+1}$ and moreover for all $0 \leq k_{1} \leq k_{2}$

$$
\sup _{s<\lambda_{t_{k_{1}}}^{k_{1}}} d\left(x_{\left(\lambda^{k_{1}}\right)_{s}^{-1}}^{k_{1}}, x_{\left(\lambda^{k_{2}}\right)_{s}^{-1}}^{k_{2}}\right)=\sup _{s<t_{k_{1}}} d\left(x_{s}^{k_{1}}, x_{\mu^{k_{1}-1} \circ \ldots \circ \mu^{k_{1}}(s)}^{k_{2}}\right) \leq \sum_{\ell=k_{1}}^{k_{2}-1} \sup _{s<\widetilde{t}_{\ell}} d\left(x_{s}^{\ell}, x_{\mu_{s}^{\ell+1}}^{\ell+1}\right) .
$$

By using (4.2) and (4.3) we get $\sum_{\ell \geq 0} \sup _{s<\widetilde{t}_{\ell}} d\left(x_{s}^{\ell}, x_{\mu_{s}^{\ell+1}}^{\ell}\right)<\infty$. By using the completeness of $(S, d)$, we deduce that, for each $m \in \mathbb{N}$, the sequence $x_{\left(\lambda^{k}\right)^{-1}}^{k}$ converges uniformly on $\left[0, \lambda_{t_{m}}^{m}\right)$. We can define $x \in \mathbb{D}_{\exp }(S)$ by setting:

$$
\xi(x):=\lim _{k \rightarrow \infty} \lambda_{t_{k}}^{k} \quad \text { and } \quad \forall s<\xi(x), x_{s}:=\lim _{k \rightarrow \infty} x_{\left(\lambda^{k}\right)_{s}^{-1}}^{k}
$$

We see that, for all $k \geq 0$

$$
\sup _{s<\lambda_{t_{k}}^{k}} d\left(x_{\left(\lambda^{k}\right)_{s}^{-1}}^{k}, x_{s}\right)=\sup _{s<t_{k}} d\left(x_{s}^{k}, x_{\lambda_{s}^{k}}\right) \leq \sum_{\ell \geq k} \sup _{s<\widetilde{t}_{\ell}} d\left(x_{s}^{\ell}, x_{\mu_{s}^{\ell}}^{\ell+1}\right) .
$$

Step 3: we prove that the infimum in (4.4) is a minimum. Suppose that there exists $k_{0} \geq 0$ such that

$$
\forall i \geq 0, \quad t_{k_{0}}<\left(\mu^{k_{0}}\right)^{-1} \circ \cdots \circ\left(\mu^{k_{0}+i-1}\right)^{-1}\left(\widetilde{t}_{k_{0}+i}\right)
$$

and we will show that one get a contradiction. Firstly, note that for all $k \geq k_{0}$ we necessarily have $\mu_{t_{k}}^{k}=t_{k+1}$ and $t_{k}<\widetilde{t}_{k}$ so, $\lambda_{t_{k}}^{k}$ is constant equal to $\xi(x)$ and furthermore

$$
d\left(x_{t_{k}}^{k}, x_{t_{k+1}}^{k+1}\right) \leq \sup _{s<\widetilde{t}_{k}} d\left(x_{s}^{k}, x_{\mu_{s}^{k}}^{k+1}\right) .
$$

Since $\sum_{k \geq 0} \sup _{s<\widetilde{t}_{k}} d\left(x_{s}^{k}, x_{\mu_{s}^{k}}^{k+1}\right)<\infty, x_{t_{k}}^{k}$ converges to an element $a \in S$. Let $\varepsilon>0$ be arbitrary such that $K:=\overline{B(a, 3 \varepsilon)} \subset S$ is compact. Let $k_{1} \geq k_{0}$ be such that

$$
d\left(x_{t_{k_{1}}}^{k_{1}}, a\right)<\varepsilon, \quad \sum_{k \geq k_{1}} \widetilde{\rho}_{\xi(x)+4 \varepsilon, K}\left(x^{k}, x^{k+1}\right)<\frac{\varepsilon}{2}
$$

and such that (4.3) holds for all $k \geq k_{1}$ with $t=\xi(x)+4 \varepsilon$. Set

$$
s_{\ell}:=\bigwedge_{0 \leq i \leq \ell}\left(\mu^{k_{1}}\right)^{-1} \circ \cdots \circ\left(\mu^{k_{1}+i-1}\right)^{-1}\left(\widetilde{t}_{k_{1}+i}\right) .
$$


It is clear that $s_{\ell}>t_{k_{1}}$ and $s_{\ell}$ is a decreasing sequence converging to $t_{k_{1}}$ so, the set $\left\{\ell>0 \mid s_{\ell}<s_{\ell-1}\right\}$ is infinite. Let $\ell>0$ be such that $s_{\ell}<s_{\ell-1}$ and $s_{\ell}-t_{k_{1}}<\varepsilon$, then

Therefore,

$$
s_{\ell}=\left(\mu^{k_{1}}\right)^{-1} \circ \cdots \circ\left(\mu^{k_{1}+\ell-1}\right)^{-1}\left(\widetilde{t}_{k_{1}+\ell}\right)
$$

$$
\begin{aligned}
& \widetilde{t}_{k_{1}+\ell}=\mu^{k_{1}+\ell-1} \circ \cdots \circ \mu^{k_{1}}\left(s_{\ell}\right)<\mu^{k_{1}+\ell-1} \circ \cdots \circ \mu^{k_{1}}\left(t_{k_{1}}+\varepsilon\right) \\
& \leq \sum_{i=k_{1}}^{k_{1}+\ell-1}\left\|\mu^{i}-\mathrm{id}\right\|_{\widetilde{t}_{i}}+t_{k_{1}}+\varepsilon \leq \sum_{i=k_{1}}^{k_{1}+\ell-1}\left\|\mu^{i}-\mathrm{id}\right\|_{\widetilde{t}_{i}}+\left\|\lambda^{k_{1}}-\mathrm{id}\right\|_{t_{k_{1}}}+\xi(x)+\varepsilon \\
& \leq \xi(x)+\varepsilon+2 \sum_{i \geq k_{1}}\left\|\mu^{i}-\mathrm{id}\right\|_{\widetilde{t}_{i}} \leq \xi(x)+\varepsilon+4 \sum_{i \geq k_{1}} \widetilde{\rho}_{\xi(x)+4 \varepsilon, K}\left(x^{i}, x^{i+1}\right)<\xi(x)+3 \varepsilon .
\end{aligned}
$$

Furthermore $\widetilde{t}_{k_{1}+\ell}<\mu_{\widetilde{t}_{k_{1}+\ell-1}}^{k_{1}+\ell-1} \leq \xi\left(x^{k_{1}+\ell}\right)$ and

$d\left(x_{\widetilde{t}_{k_{1}+\ell}}^{k_{1}+\ell}, K^{c}\right) \wedge\left(\xi(x)+4 \varepsilon-\widetilde{t}_{k_{1}+\ell}\right)+\mathbb{1}_{\widetilde{t}_{k_{1}+\ell}<\xi\left(x^{k_{1}+\ell}\right)} \leq 2 \widetilde{\rho}_{\xi(x)+4 \varepsilon, K}\left(x^{k_{1}+\ell}, x^{k_{1}+\ell+1}\right)<\varepsilon$, so, by (4.8),

$$
d\left(x_{\widetilde{t}_{k_{1}+\ell}}^{k_{1}+\ell}, K^{c}\right)<\varepsilon
$$

and

$$
d\left(x_{s_{\ell}}^{k_{1}}, K^{c}\right) \leq d\left(x_{s_{\ell}}^{k_{1}}, x_{\widetilde{t}_{k_{1}+\ell}}^{k_{1}+\ell}\right)+d\left(x_{\widetilde{t}_{k_{1}+\ell}}^{k_{1}+\ell}, K^{c}\right)<\sum_{i=k_{1}}^{k_{1}+\ell-1} \sup _{s<\widetilde{t}_{i}} d\left(x_{s}^{i}, x_{\mu_{s}^{i}}^{i+1}\right)+\varepsilon<2 \varepsilon .
$$

Hence we have $d\left(a, x_{s_{\ell}}^{k_{1}}\right)>\varepsilon$ and $d\left(a, x_{t_{k_{1}}}^{k_{1}}\right)<\varepsilon$. Letting $\ell \rightarrow \infty$ we get a contradiction.

Step 4: fix $t \geq 0$ and $K \subset S$ a compact set: we prove that $\lim _{k \rightarrow \infty} \widetilde{\rho}_{t, K}\left(x^{k}, x\right)=0$. Taking $k$ large enough (4.3) holds and by using (4.5), (4.6) and (4.7),

$$
\begin{array}{r}
\sup _{s<t_{k}} d\left(x_{s}^{k}, x_{\lambda_{s}^{k}}\right) \leq \sum_{\ell \geq k} \sup _{s<\widetilde{t}_{\ell}} d\left(x_{s}^{\ell}, x_{\mu_{s}^{\ell+1}}^{\ell}\right) \leq 2 \sum_{\ell \geq k} \widetilde{\rho}_{t, K}\left(x^{\ell}, x^{\ell+1}\right), \\
\left\|\lambda^{k}-\mathrm{id}\right\| \leq \sum_{\ell \geq k}\left\|\mu^{\ell}-\mathrm{id}\right\|_{\widetilde{t}_{\ell}} \leq 2 \sum_{\ell \geq k} \widetilde{\rho}_{t, K}\left(x^{\ell}, x^{\ell+1}\right), \\
\left\|\log \dot{\lambda}^{k}\right\| \leq \sum_{\ell \geq k}\left\|\log \dot{\mu}^{\ell}\right\|_{\widetilde{t}_{\ell}} \leq 2 \sum_{\ell \geq k} \widetilde{\rho}_{t, K}\left(x^{\ell}, x^{\ell+1}\right) .
\end{array}
$$

Moreover by the previous step we know that the infimum in (4.4) is a minimum and so, in the present step, we set

$$
\begin{aligned}
& m:=\min \left\{\ell \geq k \mid\left(\mu^{k}\right)^{-1} \circ \cdots \circ\left(\mu^{\ell-1}\right)^{-1}\left(\widetilde{t}_{\ell}\right)=t_{k}\right\} \in \mathbb{N}, \\
& M:=\sup \left\{\ell \geq k \mid\left(\mu^{k}\right)^{-1} \circ \cdots \circ\left(\mu^{\ell-1}\right)^{-1}\left(\widetilde{t}_{\ell}\right)=t_{k}\right\} \in \mathbb{N} \cup\{\infty\} .
\end{aligned}
$$

Then

$$
\begin{aligned}
d\left(x_{t_{k}}^{k}, K^{c}\right) \wedge\left(t-t_{k}\right)_{+} \mathbb{1}_{t_{k}<\xi\left(x^{k}\right)} \leq & \sum_{\ell=k}^{m-1} \sup _{s<\widetilde{t}_{\ell}} d\left(x_{s}^{\ell}, x_{\mu_{s}^{\ell}}^{\ell+1}\right) \vee\left\|\mu^{\ell}-\mathrm{id}\right\|_{\widetilde{t}_{\ell}} \\
& +d\left(x_{\widetilde{t}_{m}}^{m}, K^{c}\right) \wedge\left(t-\widetilde{t}_{m}\right)_{+} \mathbb{1}_{\widetilde{t}_{m}<\xi\left(x^{m}\right)} \\
\leq & 2 \sum_{\ell \geq k} \widetilde{\rho}_{t, K}\left(x^{\ell}, x^{\ell+1}\right) .
\end{aligned}
$$


It is clear that $\lambda_{t_{k}}^{k}=\xi(x)$ if and only if $M=\infty$. If $M<\infty$

$$
\begin{aligned}
d\left(x_{\lambda_{t_{k}}^{k}}, K^{c}\right) & \wedge\left(t-\lambda_{t_{k}}^{k}\right)_{+}=d\left(x_{\lambda_{t_{M}}^{M}}, K^{c}\right) \wedge\left(t-\lambda_{t_{M}}^{M}\right)_{+} \\
& \leq d\left(x_{\mu_{t_{M}}^{M+1}}^{M+1}, K^{c}\right) \wedge\left(t-\mu_{t_{M}}^{M}\right)_{+}+\sum_{\ell>M} \sup _{s<\widetilde{t}_{\ell}} d\left(x_{s}^{\ell}, x_{\mu_{s}^{\ell}}^{\ell+1}\right) \vee\left\|\mu^{\ell}-\mathrm{id}\right\|_{\widetilde{t}_{\ell}} \\
& \leq 2 \sum_{\ell \geq k} \widetilde{\rho}_{t, K}\left(x^{\ell}, x^{\ell+1}\right) .
\end{aligned}
$$

We have proved that

$$
\widetilde{\rho}_{t, K}\left(x^{k}, x\right) \leq 2 \sum_{\ell \geq k} \widetilde{\rho}_{t, K}\left(x^{\ell}, x^{\ell+1}\right) \underset{k \rightarrow \infty}{\longrightarrow} 0 .
$$

Step 5: we prove that $x \in \mathbb{D}_{\text {loc }}(x)$. Suppose that $\xi(x)<\infty$ and that $\left\{x_{s}\right\}_{s<\xi(x)} \Subset S$. Let $\varepsilon>0$ be such that $K:=\left\{y \in S \mid d\left(y,\left\{x_{s}\right\}_{s<\xi(x)}\right) \leq \varepsilon\right\}$ is compact and set $t=\xi(x)+\varepsilon$. By using (4.9) we have, for $k$ large enough,

$$
d\left(x_{\lambda_{t_{k}}^{k}}, K^{c}\right) \wedge\left(t-\lambda_{t_{k}}^{k}\right)_{+} \mathbb{1}_{\lambda_{t_{k}}^{k}<\xi(x)} \leq 2 \sum_{\ell \geq k} \widetilde{\rho}_{t, K}\left(x^{\ell}, x^{\ell+1}\right)<\varepsilon .
$$

Then $\xi(x)=\lambda_{t_{k}}^{k}$ and we deduce that

$$
\sup _{s<\xi(x)} d\left(x_{\left(\lambda^{k}\right)^{-1} s}^{k}, x_{s}\right) \leq 2 \sum_{\ell \geq k} \widetilde{\rho}_{t, K}\left(x^{\ell}, x^{\ell+1}\right) \underset{k \rightarrow \infty}{\longrightarrow} 0
$$

and that the limit $x_{\xi(x)-}$ exists in $S$. Therefore, $x \in \mathbb{D}_{\text {loc }}(S)$ and $x^{k}$ converges to $x$ for the local Skorokhod topology.

To prove the separability and the criterion of compactness we will use the following technical result:

Lemma 4.5. Let $R \subset S, \delta>0$ and $N \in \mathbb{N}$ be. Define

$$
\begin{array}{r}
\mathcal{E}_{R, \delta, N}:=\left\{x \in \mathbb{D}_{l o c}(S) \mid \xi(x) \leq N \delta, \forall k \in \mathbb{N}, x \text { is a constant in } R \cup\{\Delta\}\right. \\
\text { on }[k \delta,(k+1) \delta)\} .
\end{array}
$$

Then for any $x \in \mathbb{D}_{\text {loc }}(S)$

$$
\rho_{N \delta, K}\left(x, \mathcal{E}_{R, \delta, N}\right) \leq\left(\sup _{a \in K} d(a, R)+\omega_{N \delta, K, x}^{\prime}(\delta)\right) \vee \delta .
$$

Proof: For arbitrary $\varepsilon>0$, there exist $0=t_{0}<\cdots<t_{M} \leq \xi(x)$ such that

$$
\sup _{\substack{0 \leq i<M \\ t_{i} \leq s_{1}, s_{2}<t_{i+1}}} d\left(x_{s_{1}}, x_{s_{2}}\right) \leq \omega_{N \delta, K, x}^{\prime}(\delta)+\varepsilon,
$$

such that for all $0 \leq i<M, t_{i+1}>t_{i}+\delta$ and $\left(t_{M}, x_{t_{M}}\right) \notin[0, N \delta] \times K$. Denote $t^{*}:=\min \left\{s \geq 0 \mid s \geq N \delta\right.$ or $\left.d\left(x_{s}, K^{c}\right)=0\right\} \leq t_{M}$ and define $\widetilde{M}:=$ $\min \left\{0 \leq i \leq M \mid t_{i} \geq t^{*}\right\}$. Define $\widetilde{t}_{\widetilde{M}}:=\left\lceil\frac{t^{*}}{\delta}\right\rceil \delta$ where $\lceil r\rceil$ denotes the smallest integer larger or equal than the real number $r$. Moreover, for $0 \leq i<\widetilde{M}$ define 
$\widetilde{t}_{i}:=\left\lfloor\frac{t_{i}}{\delta}\right\rfloor \delta$ where we recall that $\lfloor r\rfloor$ denotes the integer part of the real number $r$, so $0=\widetilde{t}_{0}<\ldots<\widetilde{t}_{\widetilde{M}}$. Finally, we define $\widetilde{x} \in \mathcal{E}_{R, \delta, N}$ by

$$
\left\{\begin{array}{l}
\xi(\widetilde{x}):=\widetilde{t}_{\widetilde{M}}, \\
\forall 0 \leq i<M \text {, we choose } \widetilde{x}_{\widetilde{t}_{i}} \text { in } R \text { such that } d\left(x_{t_{i}}, \widetilde{x}_{\widetilde{t}_{i}}\right)<d\left(x_{t_{i}}, R\right)+\varepsilon, \\
\forall 0 \leq i<\widetilde{M}, \forall \widetilde{t}_{i} \leq s<\widetilde{t}_{i+1}: \quad \widetilde{x}_{s}:=\widetilde{x}_{\widetilde{t}_{i}},
\end{array}\right.
$$

and $\lambda \in \Lambda$ given by

We can write

$$
\left\{\begin{array}{l}
\forall 0 \leq i \leq \widetilde{M}: \quad \lambda_{t_{i} \wedge t^{*}}=\widetilde{t}_{i} \\
\forall 0 \leq i<\widetilde{M}: \quad \lambda \text { is affine on }\left[t_{i}, t_{i+1} \wedge t^{*}\right] \\
\forall s \geq t^{*}: \quad \dot{\lambda}_{s}=1
\end{array}\right.
$$

$$
\begin{aligned}
\rho_{N \delta, K}\left(x, \mathcal{E}_{R, \delta, N}\right) & \leq \rho_{N \delta, K}(x, \widetilde{x}) \leq \sup _{s<t^{*}} d\left(x_{s}, \widetilde{x}_{\lambda_{s}}\right) \vee\|\lambda-\mathrm{id}\| \\
& \leq\left(\sup _{a \in K} d(a, R)+\omega_{t, K, x}^{\prime}(\delta)+2 \varepsilon\right) \vee \delta,
\end{aligned}
$$

so, letting $\varepsilon \rightarrow 0$ we obtain the result.

The separability is an easy consequence:

Lemma 4.6. The local Skorokhod topology on $\mathbb{D}_{\text {loc }}(S)$ is separable.

Proof: Let $R$ be a countable dense part of $S$ and introduce the countable set

$$
E:=\bigcup_{n, N \in \mathbb{N}^{*}} \mathcal{E}_{R, \frac{1}{n}, N} .
$$

Consider $x \in \mathbb{D}_{\text {loc }}(S), t \geq 0, K \subset S$ a compact set and let $\varepsilon>0$ be. We choose $n \in \mathbb{N}^{*}$ such that $n^{-1} \leq \varepsilon$ and $\omega_{t+1, K, x}^{\prime}\left(n^{-1}\right) \leq \varepsilon$ and set $N:=\lceil n t\rceil$. We can write

$$
\begin{aligned}
\rho_{t, K}\left(x, \mathcal{E}_{R, \frac{1}{n}, N}\right) & \leq \rho_{\frac{N}{n}, K}\left(x, \mathcal{E}_{R, \frac{1}{n}, N}\right) \leq\left(\sup _{a \in K} d(a, R)+\omega_{\frac{N}{n}, K, x}^{\prime}\left(\frac{1}{n}\right)\right) \vee \frac{1}{n} \\
& \leq \omega_{t+1, K, x}^{\prime}\left(\frac{1}{n}\right) \vee \frac{1}{n} \leq \varepsilon .
\end{aligned}
$$

We deduce that $E$ is dense, hence $\mathbb{D}_{\text {loc }}(S)$ is separable.

We have now all the ingredients to prove the characterisation of the compactness:

Proof of Theorem 2.8: First, notice that, similarly as in the proof of Lemma 4.3, the condition (2.5) is equivalent to: for all $t \geq 0$, all compact subset $K \subset S$ and all open subset $U \subset S^{2}$ containing the diagonal $\{(y, y) \mid y \in S\}$, there exists $\delta>0$ such that for all $x \in D$ there exist $0=t_{0}<\cdots<t_{N} \leq \xi(x)$ such that

$$
\forall 0 \leq i<N, s_{1}, s_{2} \in\left[t_{i}, t_{i+1}\right), \quad\left(x_{s_{1}}, x_{s_{2}}\right) \in U,
$$

for all $0 \leq i<N, t_{i+1}-t_{i}>\delta$, and $\left(t_{N}, x_{t_{N}}\right) \notin[0, t] \times K$. Hence, the condition (2.5) is independent to $d$, and we can suppose that $(S, d)$ is complete. Suppose that $D$ satisfy condition (2.5), then, by using Lemma 4.4 , we need to prove that for all $t \geq 0, K \subset S$ a compact set and $\varepsilon>0$ arbitrary, $D$ can be recovered by a finite number of $\widetilde{\rho}_{t, K}$-balls of radius $\varepsilon$. Let $0<\eta \leq \frac{1}{9}$ be such that

$$
6 \cdot \sqrt{\eta} \vee \sup _{x \in D} \omega_{t, K, x}^{\prime}(\sqrt{\eta}) \leq \varepsilon,
$$


and let $\delta \leq \eta$ be such that

$$
\sup _{x \in D} \omega_{t+1, K, x}^{\prime}(\delta) \leq \frac{\eta}{2}
$$

Since $K$ is compact we can choose a finite set $R \subset S$ such that

$$
\sup _{a \in K} d(a, R) \leq \frac{\eta}{2}
$$

take $N:=\left\lceil t \delta^{-1}\right\rceil$. Then by using Lemma 4.5,

$$
\begin{aligned}
\sup _{x \in D} \rho_{t, K}\left(x, \mathcal{E}_{R, \delta, N}\right) & \leq \sup _{x \in D} \rho_{N \delta, K}\left(x, \mathcal{E}_{R, \delta, N}\right) \\
& \leq\left(\sup _{a \in K} d(a, R)+\sup _{x \in D} \omega_{N \delta, K, x}^{\prime}(\delta)\right) \vee \delta \leq \eta
\end{aligned}
$$

and by using Lemma 4.2,

$$
\sup _{x \in D} \widetilde{\rho}_{t, K}\left(x, \mathcal{E}_{R, \delta, N}\right) \leq 6 \sup _{x \in D}\left(\sqrt{\rho_{t, K}\left(x, \mathcal{E}_{R, \delta, N}\right)} \vee \omega_{t, K, x}^{\prime}\left(\sqrt{\rho_{t, K}\left(x, \mathcal{E}_{R, \delta, N}\right)}\right)\right) \leq \varepsilon .
$$

Since $\mathcal{E}_{R, \delta, N}$ is finite we can conclude that $D$ is relatively compact.

To prove the converse sentence, thanks to the first part of Proposition 2.7 it is enough to prove that if $x^{k}, x \in \mathbb{D}_{\text {loc }}(S)$ with $x^{k}$ converging to $x$, then for all $t \geq 0$ and all compact subset $K \subset S$,

$$
\limsup _{k \rightarrow \infty} \omega_{t, K, x^{k}}^{\prime}(\delta) \underset{\delta \rightarrow 0}{\longrightarrow} 0 .
$$

This is a direct consequence of Proposition 2.7. Let us stress that although we cite Theorem 2.4 in the proof of Proposition 2.7, in reality we only need the sequential characterisation of the convergence.

We close this section by the study of the Borel $\sigma$-algebra $\mathcal{B}\left(\mathbb{D}_{\text {loc }}(S)\right)$.

Lemma 4.7. Borel $\sigma$-algebra $\mathcal{B}\left(\mathbb{D}_{\text {loc }}(S)\right.$ ) coincides with $\mathcal{F}$.

Proof: Let $f \in \mathrm{C}\left(S^{\Delta}\right)$ and $0 \leq a<b<\infty$ be. Consider $x^{k} \in \mathbb{D}_{\text {loc }}(S)$ converging to $x \in \mathbb{D}_{\text {loc }}(S)$, with $\xi(x)>b$, and take $\lambda^{k} \in \widetilde{\Lambda}$ as in Theorem 2.4. Then, for $k$ large enough $b \vee \lambda_{b}^{k}<\xi\left(x^{k}\right)$ and by dominated convergence,

$$
\int_{a}^{b} f\left(x_{s}^{k}\right) \mathrm{d} s=\int_{a}^{\lambda_{a}^{k}} f\left(x_{s}^{k}\right) \mathrm{d} s+\int_{a}^{b} f\left(x_{\lambda_{s}^{k}}^{k}\right) \dot{\lambda}_{s}^{k} \mathrm{~d} s+\int_{\lambda_{b}^{k}}^{b} f\left(x_{s}^{k}\right) \mathrm{d} s \underset{k \rightarrow \infty}{\longrightarrow} \int_{a}^{b} f\left(x_{s}\right) \mathrm{d} s .
$$

Hence, the set $\left\{x \in \mathbb{D}_{\text {loc }}(S) \mid b<\xi(x)\right\}$ is open and on this set the function

$$
x \mapsto \int_{a}^{b} f\left(x_{s}\right) \mathrm{d} s
$$

is continuous so, for $t \geq 0$ and $\varepsilon>0$ the mapping from $\mathbb{D}_{\text {loc }}(S)$ to $\mathbb{R}$

$$
x \mapsto \begin{cases}\frac{1}{\varepsilon} \int_{t}^{t+\varepsilon} f\left(x_{s}\right) \mathrm{d} s, & \text { if } t+\varepsilon<\xi(x), \\ f(\Delta), & \text { otherwise }\end{cases}
$$

is measurable for the Borel $\sigma$-algebra $\mathcal{B}\left(\mathbb{D}_{\text {loc }}(S)\right)$ and, the same is true for the mapping $x \mapsto f\left(x_{t}\right)$, by taking the limit. Since $f$ is arbitrary, $x \mapsto x_{t}$ is also measurable and so, $\mathcal{F} \subset \mathcal{B}\left(\mathbb{D}_{\text {loc }}(S)\right)$. 
Conversely, since the space is separable, it is enough to prove that for each $x^{0} \in \mathbb{D}_{\text {loc }}(S), t \geq 0, K \subset S$ compact and $\varepsilon>0$ there exists $V \subset \mathbb{D}_{\text {loc }}(S), \mathcal{F}_{-}$ measurable, such that

$$
\left\{x \in \mathbb{D}_{\text {loc }}(S) \mid \rho_{t, K}\left(x, x^{0}\right) \leq \varepsilon\right\} \subset V \subset\left\{x \in \mathbb{D}_{\text {loc }}(S) \mid \rho_{t, K}\left(x, x^{0}\right) \leq 3 \varepsilon\right\} .
$$

Proposition 2.7 allows to get the existence of $0=t_{0}^{0}<\cdots<t_{N}^{0} \leq \xi\left(x^{0}\right)$ such that

$$
\sup _{\substack{0 \leq i<N \\ t_{i}^{0} \leq s_{1}, s_{2}<t_{i+1}^{0}}} d\left(x_{s_{1}}^{0}, x_{s_{2}}^{0}\right) \leq \varepsilon,
$$

and $\left(t_{N}^{0}, x_{t_{N}^{0}}^{0}\right) \notin[0, t] \times \in K$. If we define

$$
V:=\left\{x \in \mathbb{D}_{\mathrm{loc}}(S) \mid \begin{array}{c}
\exists 0=t_{0} \leq \cdots \leq t_{M} \leq \xi(x), M \leq N \text { such that: } \\
\forall 0 \leq i \leq M, \quad\left|t_{i}-t_{i}^{0}\right| \leq \varepsilon \\
\forall 0 \leq i<M, \quad \forall t \in\left[t_{i}, t_{i+1}\right), \quad d\left(x_{t}, x_{t_{i}^{0}}^{0}\right) \leq 2 \varepsilon \\
\\
d\left(x_{t_{M}^{0}}^{0}, K^{c}\right) \wedge\left(t-t_{M}^{0}\right)_{+} \mathbb{1}_{t_{M}^{0}<\xi\left(x^{0}\right) \leq 2 \varepsilon} \\
d\left(x_{t_{M}-}, K^{c}\right) \wedge d\left(x_{t_{M}}, K^{c}\right) \wedge\left(t-t_{M}\right)_{+} \mathbb{1}_{t_{M}<\xi(x)} \leq 3 \varepsilon
\end{array}\right\},
$$

it is straightforward to obtain (4.10). Since

$$
V=\left\{x \in \mathbb{D}_{\mathrm{loc}}(S) \mid \begin{array}{c}
\forall \delta>0, \exists 0=q_{0} \leq \cdots \leq q_{M}<\xi(x)-\delta, \quad M \leq N \text { such that: } \\
\forall 0 \leq i \leq M, \quad\left|q_{i}-t_{i}^{0}\right| \leq \varepsilon+\delta \\
\forall 0 \leq i<M, \quad \forall q \in\left[q_{i}+\delta, q_{i+1}-\delta\right], \quad d\left(x_{q}, x_{t_{i}^{0}}^{0}\right) \leq 2 \varepsilon \\
d\left(x_{t_{M}^{0}}^{0}, K^{c}\right) \wedge\left(t-t_{M}^{0}\right)_{+} \mathbb{1}_{t_{M}^{0}<\xi\left(x^{0}\right) \leq 2 \varepsilon} \\
d\left(x_{q_{M}}, K^{c}\right) \wedge\left(t-q_{M}\right)_{+} \mathbb{1}_{q_{M}<\xi(x)} \leq 3 \varepsilon+\delta
\end{array}\right\},
$$

where $q, q_{i}$ and $\delta$ are chosen to be rational, $V$ belongs $\mathcal{F}$. The proof is now complete.

\section{Acknowledgements}

The authors are grateful to the anonymous referee for his/her careful reading of the manuscript and useful suggestions.

\section{References}

P. Billingsley. Convergence of probability measures. Wiley Series in Probability and Statistics: Probability and Statistics. John Wiley \& Sons, Inc., New York, second edition (1999). ISBN 0-471-19745-9. MR1700749.

S. N. Ethier and T. G. Kurtz. Markov processes. Wiley Series in Probability and Mathematical Statistics: Probability and Mathematical Statistics. John Wiley \& Sons, Inc., New York (1986). ISBN 0-471-08186-8. MR838085.

M. Gradinaru and T. Haugomat. Lévy-type processes: convergence and discrete schemes. ArXiv Mathematics e-prints (2017a). arXiv: 1707.02889.

M. Gradinaru and T. Haugomat. Locally Feller processes and martingale local problems. ArXiv Mathematics e-prints (2017b). arXiv: 1706.04880.

O. Kallenberg. Foundations of modern probability. Probability and its Applications (New York). Springer-Verlag, New York, second edition (2002). ISBN 0-38795313-2. MR1876169.

T. G. Kurtz. Equivalence of stochastic equations and martingale problems. In Stochastic analysis 2010, pages 113-130. Springer, Heidelberg (2011). MR2789081. 
R. Rebolledo. Sur les temps d'arrêt et la topologie étroite. C. R. Acad. Sci. Paris Sér. A-B 289 (14), A707-A709 (1979). MR560339.

L. C. G. Rogers and D. Williams. Diffusions, Markov processes, and martingales. Vol. 2. Cambridge Mathematical Library. Cambridge University Press, Cambridge (2000). ISBN 0-521-77593-0. MR1780932.

D. W. Stroock. Diffusion processes associated with Lévy generators. Z. Wahrscheinlichkeitstheorie und Verw. Gebiete 32 (3), 209-244 (1975). MR0433614.

D. W. Stroock and S. R. S. Varadhan. Multidimensional diffusion processes. Classics in Mathematics. Springer-Verlag, Berlin (2006). MR2190038. 